TRANSACTIONS OF THE

AMERICAN MATHEMATICAL SOCIETY

Volume 360, Number 7, July 2008, Pages 3813-3837

S 0002-9947(08)04348-1

Article electronically published on February 13, 2008

\title{
MULTISCALE-BUMP STANDING WAVES WITH A CRITICAL FREQUENCY FOR NONLINEAR SCHRÖDINGER EQUATIONS
}

\author{
DAOMIN CAO, EZZAT S. NOUSSAIR, AND SHUSEN YAN
}

\begin{abstract}
In this paper we study the existence and qualitative property of standing wave solutions $\psi(x, t)=e^{-\frac{i E t}{\hbar}} u(x)$ for the nonlinear Schrödinger equation $i \hbar \frac{\partial \psi}{\partial t}+\frac{\hbar^{2}}{2 m} \Delta \psi-W(x) \psi+|\psi|^{p-1} \psi=0$ with $E$ being a critical frequency in the sense that $\inf _{x \in \mathbb{R}^{N}} W(x)=E$. We show that if the zero set of $V=W-E$ has $k$ isolated connected components $Z_{i}(i=1, \cdots, k)$ such that the interior of $Z_{i}$ is not empty and $\partial Z_{i}$ is smooth, $V$ has $t$ isolated zero points, $b_{i}, i=1, \cdots, t$, and $V$ has $l$ critical points $a_{i}(i=1, \cdots, l)$ such that $V\left(a_{i}\right)>0$, then for $\hbar>0$ small, there exists a standing wave solution which is trapped in a neighborhood of $\bigcup_{i=1} Z_{i} \cup\left(\bigcup_{i=1}^{t}\left\{b_{i}\right\}\right) \cup\left(\bigcup_{i=1}^{l}\left\{a_{i}\right\}\right)$. Moreover the amplitudes of the standing wave around $\bigcup_{i=1}^{k} Z_{i}, \bigcup_{i=1}^{t}\left\{b_{i}\right\}$ and $\bigcup_{i=1}^{l}\left\{a_{i}\right\}$ are of a different order of $\hbar$. This type of multi-scale solution has never before been obtained.
\end{abstract}

\section{INTRODUCTION}

The study of the Schrödinger equation is one of the main objects of quantum physics. The evolution of a group of identical particles interacting with each other in ultra-cold states, in particular, Bose-Einstein condensates, is described via Hartree approximation, to an excellent degree of accuracy by nonlinear Schrödinger equations. The equation also arises in many fields of physics. For instance, when we describe the propagation of light in some nonlinear optical materials, the nonlinear Schrödinger equations in nonlinear optics are reduced from Maxwell's equations. The nonlinear Schrödinger equation is typically the form

$$
i \hbar \frac{\partial \psi}{\partial t}=-\frac{\hbar^{2}}{2 m} \Delta \psi+W(x) \psi-|\psi|^{p-1} \psi,(t, x) \in \mathbb{R} \times \mathbb{R}^{N},
$$

where $\hbar$ denotes the Plank constant, $i$ is the imaginary unit, $1<p<\frac{N+2}{N-2}$ for $N \geq 3$ and $1<p<+\infty$ for $N=1,2$. In physical problems, a cubic nonlinearity, $p=3$, is common. In this case, the equation is called the Gross-Pitaevskii equation. In this paper, we consider the existence of standing waves of equation (1.1), namely of special solutions of the form $\psi(x, t)=e^{-\frac{i E t}{\hbar}} u(x)$, where $u(x)>0$ for $x \in \mathbb{R}^{N}$,

Received by the editors May 8, 2006 and, in revised form, June 15, 2006.

2000 Mathematics Subject Classification. Primary 35J20, 35J65.

Key words and phrases. Multiscale-bump, standing waves, nonlinear Schrödinger equation, variational method, critical point.

The first author was supported by the Fund of Distinguished Young Scholars of China and Innovative Funds of CAS in China.

The second and third authors were supported by ARC in Australia.

(C)2008 American Mathematical Society 3813

Reverts to public domain 28 years from publication 
for small $\hbar>0$. The transition from quantum mechanics to classics mechanics can be formally performed by letting $\hbar \rightarrow 0$, and thus the existence of solutions for $\hbar$ small has important physical interest. Standing waves solutions of (1.1) for small $\hbar$ are usually referred as semi-classic bound states. It is easily checked that $\psi$ of this form satisfies equation (1.1) if and only if the function $u$ satisfies

$$
\frac{\hbar^{2}}{2 m} \Delta u-(W(x)-E) u+|u|^{p-1} u=0, x \in \mathbb{R}^{N} .
$$

In this paper, we investigate problem (1.2) when

$$
\inf _{x \in \mathbb{R}^{N}} W(x)=E .
$$

Under the condition that

$$
\inf _{x \in \mathbb{R}^{N}} W(x)>E
$$

Rabinowitz proved in $[30$ that problem (1.2) has a ground state solution (mountainpass solution) for $\hbar>0$ small when $\inf _{x \in \mathbb{R}^{N}} W(x)<\liminf _{|x| \rightarrow \infty} W(x)$; furthermore, the following well-understood problem in $\mathbb{R}^{N}$ plays a crucial role in the construction of solutions of (1.2) and is considered the limiting equation for (1.2) as $\hbar \rightarrow 0$ :

$$
\Delta u-u+|u|^{p-1} u=0, x \in \mathbb{R}^{N} .
$$

On the other hand, for

$$
\inf _{x \in \mathbb{R}^{N}} W(x)<E,
$$

it is not difficult to see that problem (1.2) has no ground-state solutions (mountainpass solutions) if $\hbar>0$ is sufficiently small. In this sense, $E=\inf _{x \in \mathbb{R}^{N}} W(x)$ is called a critical frequency (or energy) for the nonlinear Schrödinger equation (1.1), or problem (1.2), in [4 by Byeon and Wang.

Under the condition $\inf _{x \in \mathbb{R}^{N}} W(x)>E$, there have been enormous investigations on problem (1.2). In [18, Floer and Weinstein considered the case $N=1$ and $p=3$. Assuming that $W$ is globally bounded, for a given nondegenerate critical point of the potential $W$, they constructed a positive solution $u_{\hbar}$ to (1.2) for small $\hbar>0$. The solutions concentrate near some critical points of $W(x)$ as $\hbar \rightarrow 0$. Their method, based on an interesting Lyapunov-Schmidt finite dimensional reduction, was extended by $\mathrm{Oh}$ in [28, 29] to include a similar result in higher dimensions, provided $1<p<\frac{N+2}{N-2}$. Other existence results for positive solutions of problem (1.2) under the condition $\inf _{x \in \mathbb{R}^{N}} W(x)>E$ can be found in 1, 2, 3, 10, 12, 13, $14,15,16,17,20,22,24,25,27,31,32$.

Uniqueness of solutions concentrating on several nondegenerate critical points of $W$ is studied by Cao and Heinz in [8. See also [11.

In all the above mentioned works, the authors use the ground state solution of the limiting equation (1.3) as a building block to construct single-bump or multibump solutions for (1.2) with each bump looking like a translated ground state solution.

It seems that Byeon and Wang [4] were the first to study energy level and the asymptotic behavior of positive solutions to problem (1.2) under the condition $\inf _{x \in \mathbb{R}^{N}} W(x)=E$. For an isolated component $A$ of $\left\{x \in \mathbb{R}^{N} \mid W(x)=E\right\}$, satisfying $\frac{x \in \mathbb{R}^{N}}{\operatorname{int}(A)}=A$, and $\operatorname{int}(A)=\bigcup_{i \in I} A_{i}$, where $\left\{A_{i}\right\}_{i \in I}$ are connected components of 
$\operatorname{int}(A)$, they proved in [4] that there is a solution $u_{\hbar}$ such that $\left(\frac{\sqrt{2 m}}{\hbar}\right)^{\frac{2}{p-1}} u_{\hbar}(x)$ converges to a function $w$ as $\hbar \rightarrow 0$, where $w=0$ for $x \in \mathbb{R}^{N} \backslash \Omega$, and $\left.w\right|_{\Omega}$ is a ground state (least energy solution) of

$$
\begin{cases}\Delta u+u^{p}=0 & x \in \Omega, \\ u>0 & \text { on } \Omega, \\ u=0 & \text { on } \partial \Omega .\end{cases}
$$

$\Omega$ is one of $A_{i}$ satisfying

$$
\begin{aligned}
& \inf \left\{\frac{1}{2} \int_{\Omega}|\nabla u|^{2}-\left.\frac{1}{p+1} \int_{\Omega}|u|^{p+1}\left|u \in H_{0}^{1}(\Omega) \backslash\{0\}, \int_{\Omega}\right| \nabla u\right|^{2}=\int_{\Omega}|u|^{p+1}\right\} \\
& =\inf \left\{c_{i} \mid i \in I\right\}
\end{aligned}
$$

with $c_{i}$ defined by

$$
\begin{aligned}
& c_{i}=\inf \left\{\frac{1}{2} \int_{A_{i}}|\nabla u|^{2}-\frac{1}{p+1} \int_{A_{i}}|u|^{p+1} \mid u \in H_{0}^{1}\left(A_{i}\right) \backslash\{0\},\right. \\
&\left.\qquad \int_{A_{i}}|\nabla u|^{2}=\int_{A_{i}}|u|^{p+1}\right\} .
\end{aligned}
$$

It is easy to see that the height of the bump for $u_{\hbar}$ tends to zero as $\hbar \rightarrow 0$. This is in striking contrast to the case $\inf _{x \in \mathbb{R}^{N}} W(x)>E$.

In [4], Byeon and Wang also proved the existence of solutions concentrating on an isolated zero point $b$ of $V(x)$. Their result shows that the height of the bump of the solution concentrating on $b$ and that of the solution concentrating on $\Omega$ are of different order.

Results on the existence of localized solutions of [4] are extended in 5 to more general nonlinearities.

The solutions obtained in [4 have one bump. In 9], Cao and Noussair obtained the existence of solutions concentrating simultaneously on several sets of $\left\{A_{i} \mid i \in\right.$ $I\}$, that is, solutions $u_{\hbar}$ such that $\hbar^{-\frac{2}{p-1}} u_{\hbar}(x) \rightarrow w$ as $\hbar \rightarrow 0$ and $w$ satisfies $w=0$ for $\mathbb{R}^{N} \backslash\left(\bigcup_{j=1}^{k} \Omega_{j}\right)$ with $\Omega_{j}$ being one set of $\left\{A_{i} \mid i \in I\right\}$ and $\left.w\right|_{\Omega_{j}}(j=1, \cdots, k)$ a positive solution of (1.4) with $\Omega$ replaced by $\Omega_{j}$ provided $\hbar$ is small enough. The energy of $u_{\hbar}$ is approximately $\hbar^{\frac{2(p+1)}{p-1}} \sum_{i=1}^{k} c_{i}$, where $c_{i}$ is defined by (1.5) with $A_{i}$ replaced by $\Omega_{i}$. Although the solution $u_{\hbar}$ has several bumps, the height of the bumps are of the same order.

The aim of this paper is to construct solutions such that they have several bumps in $\mathbb{R}^{N}$ and the heights of the bumps are of different order. In order to state the result precisely, we need to give some notation first.

Set $V(x)=W(x)-E, \varepsilon=\frac{\hbar}{\sqrt{2 m}}$. Then (1.2) becomes

$$
\left\{\begin{array}{l}
-\varepsilon^{2} \Delta u+V(x) u=|u|^{p-1} u=0, \quad x \in \mathbb{R}^{N}, \\
\lim _{|x| \rightarrow \infty} u(x)=0,
\end{array}\right.
$$

where $p \in\left(1, \frac{N+2}{N-2}\right)$ for $N \geq 3$, and $p \in(1,+\infty)$ for $N=1,2$.

For any given set $A \subset \mathbb{R}^{N}, r>0$, define $A^{r}=\left\{x \in \mathbb{R}^{N} \mid \operatorname{dist}(x, A) \leq r\right\}$ and $A_{\varepsilon}^{r}=\left\{x \in \mathbb{R}^{N} \mid \varepsilon x \in A^{r}\right\}$. 
In this paper, we always assume that the potential $V$ satisfies the following conditions:

(V1) $V \in C^{2, \alpha}\left(\mathbb{R}^{N}\right), \inf _{x \in \mathbb{R}^{N}} V(x)=0,0<V_{0}=\lim \inf _{|x| \rightarrow \infty} V(x)$.

Let

$$
\mathcal{Z}=\left\{x \in \mathbb{R}^{N} \mid V(x)=0\right\} .
$$

The potential $V(x)$ may have different behavior near different points in $\mathcal{Z}$. In this paper, we are particularly interested in the following two cases.

The first case is that $\mathcal{Z}$ contains some connected components with nonempty interior. More precisely, we assume that

(V2-1) $\mathcal{Z}$ has $k$ isolated components $\Omega_{i}(i=1, \cdots, k)$ such that the interior of each $\Omega_{i}$, denoted by $\operatorname{int}\left(\Omega_{i}\right)$, is not empty, and $\Omega_{i}=\operatorname{int}\left(\Omega_{i}\right)$. Furthermore, for some $r>0, \Omega_{i}^{8 r} \cap\left(\mathcal{Z} \backslash \Omega_{i}\right)=\emptyset$

(V2-2) For each $\Omega_{i}(i=1, \cdots, k)$, the problem

$$
\left\{\begin{array}{l}
-\Delta u=|u|^{p-1} u, \quad x \in \Omega_{i} \\
u(x)=0, \quad \partial \Omega_{i}
\end{array}\right.
$$

has a unique positive solution $W_{i}$. Moreover $W_{i}$ is nondegenerate, that is,

$$
\left\{\begin{array}{l}
-\Delta u=p W_{i}^{p-1} u, \quad x \in \Omega_{i}, \\
u(x)=0, \quad x \in \partial \Omega_{i},
\end{array}\right.
$$

has only a trivial solution $u \equiv 0$.

Remark 1.1. If $\Omega_{i}$ is a ball for each $i=1, \cdots, k$, then (V2-2) holds (see 21]). If $p$ is close to $\frac{N+2}{N-2}$ for $N \geq 3$, then by a result of Grossi [20, (V2 - 2) holds if $\Omega_{i}$ is convex and symmetric with respect to the coordinate plane.

The second case is that $\mathcal{Z}$ contains some isolated zero points $\left\{b_{1}, \cdots, b_{t}\right\}$ of $V(x)$. We assume

(V3) There are points $b_{i} \in \mathcal{Z}, i=1, \cdots, t$, such that

$$
V(x)=\left|x-b_{i}\right|^{m_{i}}+Q\left(x-b_{i}\right) \quad \text { for } x \text { close to } b_{i},
$$

where $m_{i}>0$ is a real number, $\lim _{|x| \rightarrow 0} Q(x)|x|^{-m_{i}}=0$.

By a result of [7, the problem

$$
\left\{\begin{array}{l}
-\Delta u+|x|^{m_{i}} u=u^{p}, \quad x \in \mathbb{R}^{N}, \\
\lim _{|x| \rightarrow \infty} u(x)=0
\end{array}\right.
$$

has a unique positive solution $\widehat{W}_{m_{i}}$. Furthermore, $\widehat{W}_{m_{i}}$ is nondegenerate, that is,

$$
\left\{\begin{array}{l}
-\Delta u+|x|^{m_{i}} u=p \widehat{W}_{m_{i}}^{p-1} u, \quad x \in \mathbb{R}^{N} \\
\lim _{|x| \rightarrow \infty} u(x)=0
\end{array}\right.
$$

has only a trivial solution $u \equiv 0$.

Let us recall some known results about (1.6). When (V1) (V2-1) are satisfied, then for each $\Omega_{i}$, by a result of Byeon and Wang [4, (1.6) has a solution $W_{\varepsilon, i}$ for 
$\varepsilon$ small. $W_{\varepsilon, i}$ satisfies that as $\varepsilon \rightarrow 0$,

$$
\begin{gathered}
\varepsilon^{-\frac{2}{p-1}} W_{\varepsilon . i}(x) \rightarrow W_{i}(x) \text { in } C^{1, \alpha}\left(\Omega_{i}\right) \text { and } H^{1}\left(\mathbb{R}^{N}\right), \\
W_{\varepsilon, i}(x) \leq C e^{-\gamma_{0} \operatorname{dist}\left(x, \Omega_{i}\right) / \varepsilon} \text { for } x \in \mathbb{R}^{N}, \\
\int_{\mathbb{R}^{N}}\left(\left|\nabla W_{\varepsilon, i}\right|^{2}+W_{\varepsilon, i}^{p+1}\right) \leq C \varepsilon^{\frac{2(p+1)}{p-1}},
\end{gathered}
$$

where $C>0, \gamma_{0}>0$ are some constants. Here, we assume that $W_{i}=0$ in $\mathbb{R}^{N} \backslash \Omega_{i}$.

When $V$ satisfies (V1)-(V3), following a result of Byeon and Wang [4, (1.6) has a positive solution $\widetilde{W}_{\varepsilon, i}(x)$. As $\varepsilon \rightarrow 0, \widetilde{W}_{\varepsilon, i}(x)$ satisfies

$$
\widehat{W}_{\varepsilon, i}(x)=: \varepsilon^{-\frac{2 m_{i}}{(p-1)\left(m_{i}+2\right)}} \widetilde{W}_{\varepsilon, i}\left(\varepsilon^{\frac{2}{m_{i}+2}} x+b_{i}\right) \rightarrow \widehat{W}_{m_{i}}(x), \quad \text { in } H^{1}\left(\mathbb{R}^{N}\right),
$$

where $\widehat{W}_{m_{i}}$ is the positive solution of (1.8). Furthermore

$$
\begin{gathered}
\widetilde{W}_{\varepsilon, i}(x) \leq C e^{-\gamma_{1}\left|x-b_{i}\right| / \varepsilon} \quad \text { for } x \in \mathbb{R}^{N}, \\
\int_{\mathbb{R}^{N}}\left(\left|\nabla \widetilde{W}_{\varepsilon, i}\right|^{2}+\widetilde{W}_{\varepsilon, i}^{p+1}\right) \leq C \varepsilon^{\frac{2 m_{i}(p+1)}{(p-1)\left(m_{i}+2\right)}+\frac{2 N}{m_{i}+2}},
\end{gathered}
$$

where $C>0$ and $\gamma_{1}>0$ are some constants.

Since $V(x) \geq 0$, every point in $\mathcal{Z}$ is a critical point of $V$. Of course, $V$ may have critical points, where $V$ is positive. We assume

(V4) There are $\left\{a_{1}, \cdots, a_{l}\right\}$, such that $V\left(a_{i}\right)>0, i=1, \cdots, l$, and $a_{i}$ is a nondegenerate critical point of $V, i=1, \cdots, l$. That is, the matrix $\left(\nabla^{2} V\left(a_{i}\right)\right)$ is invertible.

It is well known that

$$
\left\{\begin{array}{l}
-\Delta u+V\left(a_{i}\right) u=u^{p}, \quad x \in \mathbb{R}^{N}, \\
u(x) \rightarrow 0 \quad \text { as }|x| \rightarrow \infty
\end{array}\right.
$$

has a unique positive solution which will be denoted by $U_{i}(x) . U_{i}(x)$ is a radial, decreasing function and satisfies

$$
\left\{\begin{array}{l}
U_{i}(x) e^{\sqrt{V\left(a_{i}\right)}|x|}|x|^{\frac{N-1}{2}} \rightarrow c_{i}>0, \\
\nabla U_{i}(x) \cdot \frac{x}{|x|} e^{\sqrt{V\left(a_{i}\right)}|x|}|x|^{\frac{N-1}{2}} \rightarrow-c_{i},
\end{array}\right.
$$

as $|x| \rightarrow+\infty$.

Define the inner product of $H^{1}\left(\mathbb{R}^{N}\right)$ by

$$
\langle u, v\rangle_{\varepsilon}=\int_{\mathbb{R}^{N}}\left(\varepsilon^{2} \nabla u \nabla v+V(x) u v\right)
$$

and the norm induced by $\langle\cdot, \cdot\rangle_{\varepsilon}$ is denoted by $\|\cdot\|_{\varepsilon}$.

We will use the convention that if $l=0$, then the summation from 0 to $l$ is zero. Now we are ready to state our main results.

Theorem 1.1. Suppose that (V1) holds. Let $\Omega_{1}, \cdots, \Omega_{k}$ be the connected components of $\mathcal{Z}$, such that $(\mathbf{V} \mathbf{2}-\mathbf{1})$ and $(\mathbf{V} \mathbf{2}-\mathbf{2})$ hold. Let $b_{1}, \cdots, b_{t}$ be the points such that (V3) holds. Let $a_{1}, \cdots, a_{l}$ be the points such that (V4) holds. Then for $\varepsilon$ small, (1.6) has a positive solution $u_{\varepsilon}$ such that

$$
u_{\varepsilon}(x)=\sum_{i=1}^{k} W_{\varepsilon, i}(x)+\sum_{i=1}^{t} \widetilde{W}_{\varepsilon, i}+\sum_{i=1}^{l} U\left(\frac{x-y_{\varepsilon, i}}{\varepsilon}\right)+\omega_{\varepsilon}(x),
$$


where $\omega_{\varepsilon} \in H^{1}\left(\mathbb{R}^{N}\right)$ satisfying

$$
\left|\omega_{\varepsilon}(x)\right| \leq C e^{-\sigma / \varepsilon}, \quad x \in \mathbb{R}^{N} \backslash \bigcup_{i=1}^{l} B_{\delta}\left(a_{i}\right),
$$

for some small constants $\sigma>0$ and $\delta>0$,

$$
\begin{gathered}
\left\|\omega_{\varepsilon}\right\|_{\varepsilon}=O\left(\varepsilon^{\frac{N}{2}+2}\right), \quad\left\|\omega_{\varepsilon}\right\|_{L^{\infty}}=o(1), \\
y_{\varepsilon, i} \rightarrow a_{i}, \quad i=1, \cdots, l, \quad \text { as } \quad \varepsilon \rightarrow 0 .
\end{gathered}
$$

By the same argument for the proof of Theorem 1.1. we can prove the following special case (corresponding to $k=t=0$ in Theorem 1.1).

Theorem 1.2. Suppose that (V1) holds. Let $a_{1}, \cdots, a_{l}$ be the points such that (V4) holds. Then for $\varepsilon$ small, (1.6) has a positive solution $u_{\varepsilon}$ such that

$$
u_{\varepsilon}(x)=\sum_{i=1}^{l} U\left(\frac{x-y_{\varepsilon, i}}{\varepsilon}\right)+\omega_{\varepsilon}(x),
$$

where $\omega_{\varepsilon} \in H^{1}\left(\mathbb{R}^{N}\right)$ satisfying (1.17), (1.18) and (1.19) in Theorem 1.1 .

By (1.9), (1.12), (1.16) and (1.17), $u_{\varepsilon}$ obtained in Theorem 1.1 is multi-scale, because $u_{\varepsilon}$ is approximately of level $\varepsilon^{\frac{2}{p-1}}$ around $\Omega_{i}$, is approximately of level $\varepsilon^{\frac{2 m_{i}}{(p-1)\left(m_{i}+2\right)}}$ around $b_{i}$, and $u_{\varepsilon}$ has a positive lower bound independent of $\varepsilon$ around $a_{i}$ for small $\varepsilon$. Therefore the solution obtained in Theorem 1.1 takes the profile of $W_{\varepsilon, i}, \tilde{W}_{\varepsilon, i}$ and $U\left(\frac{x-y_{\varepsilon, i}}{\varepsilon}\right)$ respectively near the corresponding domains or points.

Let us point out that the result in Theorem 1.2 , which generalizes the corresponding results of $\mathrm{Oh}$ in [28] and 29] to nonnegative potentials (a uniform positive lower bound is needed in [28 and 29]), is new. The proof of this result does not follow the reduction procedure in a standard way, because $\|u\|_{\varepsilon}$ cannot control the $L^{p}$ norm of $u$ uniformly if $\mathcal{Z}$ is not empty.

The only known results on the existence of solutions with multi-scale were obtained by Byeon and Oshita in [6], where they require $N \leq 5$ and only consider the cases that $p=2,3$ or $p \geq 4$ for $N=1,2, p=2,3$ or $4 \leq p<5$ for $N=3$ and $p=2$ for $N=4,5$. In the present paper, we consider the case $N \geq 1$ and allow $1<p<\frac{N+2}{N-2}$ for $N \geq 3,1<p<+\infty$ for $N=1$, 2. Although in principle the method used in [6] and that used in the present paper is similar, the way of constructing the solutions is different. Roughly speaking, taking $k=1, l=1$ and $i=1$ for instance, in 6 the main terms of solution were taken as

$$
w_{\varepsilon, y}=W_{\varepsilon, 1}+\tilde{W}_{\varepsilon, 1}+U\left(\frac{x-y}{\varepsilon}\right)+\sum_{i=1}^{4} \varepsilon^{i} U_{i, y}(x),
$$

where $U_{i, y}$ are deduced inductively. The correction term $v_{\varepsilon, y}$ is then obtained so that $w_{\varepsilon, y}+v_{\varepsilon, y}$ is a real solution. To analyze the interaction between bumps and to obtain estimates for $v_{\varepsilon, y}$, expansion of $f(s)=|s|^{p-1} s$ up to the fourth order is used. This requires that $f \in C^{4}(\mathbb{R})$ (see page 1894 in [6]) and therefore $p$ is assumed to satisfy that either $p$ is an integer or $4 \leq p<5$. To make sure that the solution obtained is nonnegative, they need to show that the $L^{2}$ norm of the negative part of the solution is sufficiently small provided it is not identically zero. To obtain this, $p \geq 2$ is assumed which requires that $N \leq 5$. In our paper we take $f(s)=s_{+}^{p}$ 
and we use a different way of constructing solutions. To be more precise, we take the main terms of the solution in the following way:

$$
w_{\varepsilon, y}=W_{\varepsilon, 1}+\tilde{W}_{\varepsilon, 1}+U\left(\frac{x-y}{\varepsilon}\right) .
$$

Then we try to obtain a corresponding correction term $\omega_{\varepsilon}$ in a subset with small $H^{1}$ and $L^{\infty}$ norm. Furthermore $\omega_{\varepsilon}$ should be exponentially small in $\Omega_{1}$ and near $b_{1}$ (see (1.17) and (1.18) $)$, which is necessary to guarantee that $w_{\varepsilon, y_{\varepsilon}}+\omega_{\varepsilon}$ is really a solution with multi-scale bumps. To obtain existence of such a correction term is the key point, and we take a lot of efforts to accomplish this.

We end this section by giving the outline of our proof of the main results.

For the simplicity of the notation we only prove the case $k=t=l=1$. In this case, we drop all the subscripts $i$ in Theorem 1.1. Thus, Theorem 1.1 can be stated as

Theorem 1.3. Suppose that $k=t=l=1$. Assume (V1), (V2), (V3) and (V4) hold. Then for $\varepsilon$ small, (1.6) has a solution $u_{\varepsilon}$ such that

$$
u_{\varepsilon}(x)=W_{\varepsilon}(x)+\widetilde{W}_{\varepsilon}(x)+U\left(\frac{x-y_{\varepsilon}}{\varepsilon}\right)+\omega_{\varepsilon}(x),
$$

where $\omega_{\varepsilon}(x)$ and $y_{\varepsilon}$ satisfy (1.17), (1.18) and (1.19) in Theorem 1.1.

Let us give some notation.

Define $U_{\varepsilon, y}(x)=U\left(\frac{x-y}{\varepsilon}\right)$,

$$
\begin{gathered}
E_{\varepsilon, y}=\left\{\omega \in H^{1}\left(\mathbb{R}^{N}\right) \quad \mid\left\langle\omega, \frac{\partial U_{\varepsilon, y}}{\partial x_{i}}\right\rangle_{\varepsilon}=0, i=1, \cdots, N\right\}, \\
\Sigma_{\varepsilon, \delta, \sigma}=\left\{(y, \omega) \quad \mid \quad y \in B_{\delta}\left(a_{1}\right), \omega \in E_{\varepsilon, y},\|\omega\|_{\varepsilon} \leq \varepsilon^{\frac{N}{2}+\sigma}\right\}, \\
\Phi_{\varepsilon}(x)=W_{\varepsilon}(x)+\widetilde{W}_{\varepsilon}(x) .
\end{gathered}
$$

To prove Theorem 1.3, we will construct a solution of the form $u_{\varepsilon}=\Phi_{\varepsilon}(x)+u$, with $u$ satisfying

$$
\left\{\begin{array}{l}
-\varepsilon^{2} \Delta u+V(x) u=u_{+}^{p}+g_{\varepsilon}(x, u), \quad x \in \mathbb{R}^{N}, \\
u(x) \rightarrow 0 \text { as }|x| \rightarrow \infty
\end{array}\right.
$$

where

$$
g_{\varepsilon}(x, t)=\left(\Phi_{\varepsilon}(x)+t\right)_{+}^{p}-W_{\varepsilon}^{p}(x)-\widetilde{W}_{\varepsilon}^{p}(x)-t_{+}^{p} .
$$

Set

$$
\begin{gathered}
G_{\varepsilon}(x, t)=\int_{0}^{t} g_{\varepsilon}(x, \tau) d \tau \\
I(u)=\frac{1}{2} \int_{\mathbb{R}^{N}}\left(\varepsilon^{2}|\nabla u|^{2}+V(x) u^{2}\right)-\frac{1}{p+1} \int_{\mathbb{R}^{N}} u_{+}^{p+1}-\int_{\mathbb{R}^{N}} G_{\varepsilon}(x, u),
\end{gathered}
$$

and

$$
J(y, \omega)=I\left(U_{\varepsilon, y}+\omega\right) \quad \text { for } \quad(y, \omega) \in \Sigma_{\varepsilon, \delta, \sigma} .
$$

It is known that for $\delta$ small $u_{\varepsilon}=U_{\varepsilon, y}+\omega$ is a critical point of $I$ in $H^{1}\left(\mathbb{R}^{N}\right)$ if and only if $(y, \omega)$ is a critical point of $J$. A point $(y, \omega)$ is a critical point of $J$ if 
and only if there exist $D_{j} \in \mathbb{R}(j=1, \cdots, N)$ such that

$$
\begin{gathered}
\left\langle\frac{\partial J}{\partial \omega}, \varphi\right\rangle=\sum_{j=1}^{N} D_{j}\left\langle\frac{\partial U_{\varepsilon, y}}{\partial x_{j}}, \quad \varphi\right\rangle_{\varepsilon} \quad \forall \varphi \in H^{1}\left(\mathbb{R}^{N}\right), \\
\frac{\partial J}{\partial y_{j}}=\sum_{i=1}^{N} D_{i}\left\langle\frac{\partial^{2} U_{\varepsilon, y}}{\partial x_{i} \partial x_{j}}, \omega\right\rangle_{\varepsilon} .
\end{gathered}
$$

Our strategy in proving Theorem 1.3 is first to find, for given $y \in B_{\delta}\left(a_{1}\right)$, $\omega_{\varepsilon, y} \in E_{\varepsilon, y}$ such that (1.22) is satisfied provided $\delta$ and $\varepsilon$ are small. Then we try to obtain a suitable point $y_{\varepsilon}$ in $B_{\delta}\left(a_{1}\right)$ so that (1.23) is satisfied as well. The main difficulties in the proof of the result are the following. First, we try to glue together bumps of different scales. To make sure that $\omega_{\varepsilon}$ is a small term of the all the bumps, we need to prove that the infinite norm of $\omega_{\varepsilon}$ is of higher order term of the heights of all the bumps. Second, as we pointed out earlier, if $\mathcal{Z}$ is not empty, the norm $\|u\|_{\varepsilon}$ cannot control the $L^{p}$ norm of $u$ uniformly. So we cannot carry out the reduction procedure in a standard way. In this paper, we achieve the first step by carrying out the reduction argument in a carefully chosen subset of $E_{\varepsilon, y}$, and we finish the second step by using a degree argument.

\section{THE REDUCTION}

As indicated in section 2, we will consider the positive solution of

$$
\left\{\begin{array}{l}
-\varepsilon^{2} \Delta u+V(x) u=u_{+}^{p}+g_{\varepsilon}(x, u), \quad x \in \mathbb{R}^{N}, \\
u(x) \rightarrow 0 \text { as }|x| \rightarrow \infty .
\end{array}\right.
$$

We will reduce the problem of finding a solution for (2.1) concentrating in near $a_{1}$ to a finite dimensional problem this section.

In what follows, we assume that $b_{1}=0$.

Expanding $J(y, \omega)$ at $\omega \equiv 0$, we get

$$
J(y, \omega)=J(y, 0)+\ell_{\varepsilon}(\omega)+\frac{1}{2} Q_{\varepsilon}(\omega, \omega)+R_{\varepsilon}(\omega)
$$

with

$$
\begin{gathered}
\ell_{\varepsilon}(\omega)=\left\langle U_{\varepsilon, y}, \omega\right\rangle_{\varepsilon}-\int_{\mathbb{R}^{N}} U_{\varepsilon, y}^{p} \omega-\int_{\mathbb{R}^{N}} g_{\varepsilon}\left(x, U_{\varepsilon, y}\right) \omega, \\
Q_{\varepsilon}(\omega, \eta)=\langle\omega, \eta\rangle_{\varepsilon}-p \int_{\mathbb{R}^{N}} U_{\varepsilon, y}^{p-1} \omega \eta-\int_{\mathbb{R}^{N}} g_{\varepsilon}^{\prime}\left(x, U_{\varepsilon, y}\right) \omega \eta
\end{gathered}
$$

for $\omega, \eta \in E_{\varepsilon, y}$,

$$
\begin{aligned}
R_{\varepsilon}(\omega)= & \frac{1}{p+1} \int_{\mathbb{R}^{N}}\left[\left(U_{\varepsilon, y}+\omega\right)_{+}^{p+1}-U_{\varepsilon, y}^{p+1}-(p+1) U_{\varepsilon, y}^{p} \omega-\frac{1}{2}(p+1) p U_{\varepsilon, y}^{p-1} \omega^{2}\right] \\
& +\int_{\mathbb{R}^{N}}\left[G_{\varepsilon}\left(x, U_{\varepsilon, y}+\omega\right)-G_{\varepsilon}\left(x, U_{\varepsilon, y}\right)-g_{\varepsilon}\left(x, U_{\varepsilon, y}\right) \omega-\frac{1}{2} g_{\varepsilon}^{\prime}\left(x, U_{\varepsilon, y}\right) \omega^{2}\right] .
\end{aligned}
$$

Here and in what follows we always denote $g_{\varepsilon}^{\prime}(x, t)=\frac{\partial g_{\varepsilon}(x, t)}{\partial t}$.

Our first preliminary result is the following

Lemma 2.1. $\ell_{\varepsilon}(\omega)$ is a bounded linear functional and there exists a constant $C>0$ such that

$$
\left|\ell_{\varepsilon}(\omega)\right| \leq C\left(\left|V(y)-V\left(x_{0}\right)\right|+\varepsilon|\nabla V(y)|+\varepsilon^{2}\right) \varepsilon^{\frac{N}{2}}\|\omega\|_{\varepsilon} .
$$


Proof. From the equation satisfied by $U_{\varepsilon, y}$, we have

$$
\ell_{\varepsilon}(\omega)=\int_{\mathbb{R}^{N}}\left(V(x)-V\left(x_{0}\right)\right) U_{\varepsilon, y} \omega-\int_{\mathbb{R}^{N}} g_{\varepsilon}\left(x, U_{\varepsilon, y}\right) \omega .
$$

For the first term in (2.7), using the estimate

$$
\int_{\mathbb{R}^{N}} \omega^{2} \leq C \int_{\mathbb{R}^{N}}\left(|\nabla \omega|^{2}+V(x) \omega^{2}\right) \leq C \varepsilon^{-2}\|\omega\|_{\varepsilon}^{2},
$$

we have

$$
\begin{aligned}
& \left|\int_{\mathbb{R}^{N}}\left(V(x)-V\left(x_{0}\right)\right) U_{\varepsilon, y} \omega\right| \\
& =\left|\left(\int_{B_{\delta}(y)}+\int_{\mathbb{R}^{N} \backslash B_{\delta}(y)}\right)\left(V(x)-V\left(x_{0}\right)\right) U_{\varepsilon, y} \omega\right| \\
& \leq\left|V(y)-V\left(x_{0}\right)\right|\left(\int_{B_{\delta}(y)} U_{\varepsilon, y}^{2}\right)^{\frac{1}{2}}\left(\int_{B_{\delta}(y)} \omega^{2}\right)^{\frac{1}{2}} \\
& \quad+\left|\int_{B_{\delta}(y)}(V(x)-V(y)) U_{\varepsilon, y} \omega\right|+C e^{-\gamma \delta / \varepsilon}\left(\int_{\mathbb{R}^{N}} \omega^{2}\right)^{\frac{1}{2}} \\
& \leq C \varepsilon^{\frac{N}{2}}\left(\left|V(y)-V\left(x_{0}\right)\right|+\varepsilon|\nabla V(y)|+\varepsilon^{2}\right)\|\omega\|_{\varepsilon},
\end{aligned}
$$

where $\gamma>0$ is some constant.

On the other hand,

$$
\begin{aligned}
& \left|\int_{\mathbb{R}^{N}} g_{\varepsilon}\left(x, U_{\varepsilon, y}\right) \omega\right| \\
& \leq C \int_{\mathbb{R}^{N}}\left[W_{\varepsilon}^{p-1}\left(U_{\varepsilon, y}+\widetilde{W}_{\varepsilon}\right)+U_{\varepsilon, y}^{p-1}\left(W_{\varepsilon}+\widetilde{W}_{\varepsilon}\right)+\widetilde{W}_{\varepsilon}^{p-1}\left(U_{\varepsilon, y}+W_{\varepsilon}\right)\right]|\omega| \\
& \leq C e^{-\gamma / \varepsilon}\|\omega\|_{\varepsilon}
\end{aligned}
$$

for some $\gamma>0$. It is easy to see that (2.6) follows from (2.8) and (2.9).

Lemma 2.2. $Q_{\varepsilon}$ is a bounded bilinear operator on $E_{\varepsilon, y}$ and there is a positive constant $C$ such that

$$
\left|Q_{\varepsilon}(\omega, \eta)\right| \leq C\|\omega\|_{\varepsilon}\|\eta\|_{\varepsilon} .
$$

Proof. With direct computations and using (1.16), we have

$$
\begin{aligned}
& \left|\int_{\mathbb{R}^{N}} U_{\varepsilon, y}^{p-1} \omega \eta\right| \\
& \leq\left|\left(\int_{B_{\delta}(y)}+\int_{\mathbb{R}^{N} \backslash B_{\delta}(y)}\right) U_{\varepsilon, y}^{p-1} \omega \eta\right| \\
& \leq\left(\int_{B_{\delta}(y)} V(x) \omega^{2}\right)^{\frac{1}{2}}\left(\int_{B_{\delta}(y)} V(x) \eta^{2}\right)^{\frac{1}{2}}+C e^{-2 \gamma / \varepsilon} \int_{\mathbb{R}^{N} \backslash B_{\delta}(y)}|\omega||\eta| \\
& \leq\left(C+C e^{-\gamma / \varepsilon}\right)\|\omega\|_{\varepsilon}\|\eta\|_{\varepsilon}
\end{aligned}
$$


and

$$
\begin{aligned}
& \left|\int_{\mathbb{R}^{N}} g_{\varepsilon}^{\prime}\left(x, U_{\varepsilon, y}\right) \omega \eta\right| \\
& \leq C\left(\int_{\mathbb{R}^{N}} W_{\varepsilon}^{p-1}|\omega||\eta|+\int_{\mathbb{R}^{N}} \widetilde{W}_{\varepsilon}^{p-1}|\omega||\eta|+\int_{\mathbb{R}^{N}} U_{\varepsilon, y}^{\frac{p-1}{2}}\left(W_{\varepsilon}+\widetilde{W}_{\varepsilon}\right)^{\frac{p-1}{2}}|\omega||\eta|\right) .
\end{aligned}
$$

It is easy to see

$$
\begin{aligned}
\int_{\mathbb{R}^{N}} W_{\varepsilon}^{p-1}|\omega||\eta| & =\varepsilon^{2} \int_{\mathbb{R}^{N}}\left(\varepsilon^{-\frac{2}{p-1}} W_{\varepsilon}\right)^{p-1}|\omega||\eta| \\
& \leq C \varepsilon^{2}\left(\int_{\mathbb{R}^{N}}|\omega|^{2}\right)^{\frac{1}{2}}\left(\int_{\mathbb{R}^{N}}|\eta|^{2}\right)^{\frac{1}{2}} \\
& \leq C\|\omega\|_{\varepsilon}\|\eta\|_{\varepsilon} .
\end{aligned}
$$

Denote $\widetilde{\omega}(x)=\omega\left(\varepsilon^{\frac{2}{m+2}} x\right), \delta_{\varepsilon}=\varepsilon^{-\frac{2}{m+2}} \delta$. Then for $\varepsilon>0$ small,

$$
\begin{aligned}
\varepsilon^{\frac{m}{m+2}}\left(\int_{B_{\delta}(0)} \omega^{2}(x) d x\right)^{\frac{1}{2}} & =\varepsilon^{\frac{m+N}{m+2}}\left(\int_{B_{\delta_{\varepsilon}}(0)} \tilde{\omega}^{2}(\xi) d \xi\right)^{\frac{1}{2}} \\
& \leq C \varepsilon^{\frac{m+N}{m+2}}\left(\int_{B_{\delta_{\varepsilon}}(0)}\left(|\nabla \tilde{\omega}|^{2}+|\xi|^{m} \widetilde{\omega}^{2}(\xi) d \xi\right)\right)^{\frac{1}{2}} \\
& \leq C\left(\int_{B_{\delta}(0)}\left(\varepsilon^{2}|\nabla \omega|^{2}+V(x) \omega^{2}\right) d x\right)^{\frac{1}{2}} \\
& \leq C\|\omega\|_{\varepsilon} .
\end{aligned}
$$

Hence by (2.14) and (1.12),

$$
\begin{aligned}
\int_{\mathbb{R}^{N}} \widetilde{W}_{\varepsilon}^{p-1}|\omega||\eta|= & \varepsilon^{\frac{2 m}{m+2}} \int_{B_{\delta}(0)} \widehat{W}_{\varepsilon}^{p-1}\left(\varepsilon^{-\frac{2}{m+2}} x\right)|\omega(x) \| \eta(x)| d x \\
& +C e^{-2 \gamma / \varepsilon}\left(\int_{\mathbb{R}^{N} \backslash B_{\delta}(0)}|\omega|^{2}\right)^{\frac{1}{2}}\left(\int_{\mathbb{R}^{N} \backslash B_{\delta}(0)}|\eta|^{2}\right)^{\frac{1}{2}} \\
\leq & C \varepsilon^{\frac{2 m}{m+2}}\left(\int_{B_{\delta}(0)}|\omega|^{2}\right)^{\frac{1}{2}}\left(\int_{B_{\delta}(0)}|\eta|^{2}\right)^{\frac{1}{2}} \\
& +C e^{-2 \gamma / \varepsilon}\|\omega\|_{\varepsilon}\|\eta\|_{\varepsilon} \\
\leq & C\|\omega\|_{\varepsilon}\|\eta\|_{\varepsilon} .
\end{aligned}
$$

Finally,

$$
\begin{aligned}
\int_{\mathbb{R}^{N}} U_{\varepsilon, y}^{\frac{p-1}{2}}\left(W_{\varepsilon}+\widetilde{W}_{\varepsilon}\right)^{\frac{p-1}{2}}|\omega||\eta| & \leq C e^{-2 \gamma / \varepsilon}\left(\int_{\mathbb{R}^{N}}|\omega|^{2}\right)^{\frac{1}{2}}\left(\int_{\mathbb{R}^{N}}|\eta|^{2}\right)^{\frac{1}{2}} \\
& \leq C\|\omega\|_{\varepsilon}\|\eta\|_{\varepsilon} .
\end{aligned}
$$

So, (2.10) follows easily from (2.11)-(2.13), (2.15) and (2.16). 
From Lemma 2.2 we obtain the existence of a linear operator $L_{\varepsilon}: E_{\varepsilon, y} \rightarrow E_{\varepsilon, y}$ such that for any $\omega, \eta \in E_{\varepsilon, y}$

$$
\left\langle L_{\varepsilon} \omega, \eta\right\rangle_{\varepsilon}=Q_{\varepsilon}(\omega, \eta) .
$$

For the $L_{\varepsilon}$ defined as above we have

Proposition 2.3. There are $\varepsilon_{1}>0, \delta_{1}>0$ and $\rho>0$ such that for $\varepsilon \in\left(0, \varepsilon_{1}\right), \delta \in$ $\left(0, \delta_{1}\right]$ and for all $y \in B_{\delta}\left(a_{1}\right), \omega \in E_{\varepsilon, y}$

$$
\left\|L_{\varepsilon} \omega\right\|_{\varepsilon} \geq \rho\|\omega\|_{\varepsilon} .
$$

Proof. Suppose our conclusion is false. Then there exist a sequence $\left\{\varepsilon_{n}\right\}, \varepsilon_{n} \rightarrow$ $0,\left\{y_{\varepsilon_{n}}\right\} \subset \mathbb{R}^{N}, y_{\varepsilon_{n}} \rightarrow a_{1}$ and $\omega_{\varepsilon_{n}} \in E_{\varepsilon_{n}, y_{\varepsilon_{n}}}$ such that

$$
\left\|L_{\varepsilon_{n}} \omega_{\varepsilon_{n}}\right\|_{\varepsilon_{n}}=o(1)\left\|\omega_{\varepsilon_{n}}\right\|_{\varepsilon_{n}} \text {. }
$$

To simplify the notation, we write $\varepsilon_{n}$ and $\omega_{\varepsilon_{n}}$ as $\varepsilon$ and $\omega_{\varepsilon}$, respectively, and write $y_{\varepsilon_{n}}$ as $y_{\varepsilon}$. By our assumption we have

$$
\int_{\mathbb{R}^{N}}\left(\varepsilon^{2} \nabla \omega_{\varepsilon} \nabla \eta+V(x) \omega_{\varepsilon} \eta\right)-p \int_{\mathbb{R}^{N}}\left(\Phi_{\varepsilon}+U_{\varepsilon, y_{\varepsilon}}\right)^{p-1} \omega_{\varepsilon} \eta=o(1)\|\omega\|_{\varepsilon}\|\eta\|_{\varepsilon}
$$

for $\omega, \eta \in E_{\varepsilon, y_{\varepsilon}}$, that is, $\phi=\omega, \eta$ satisfies

$$
\int_{\mathbb{R}^{N}}\left(\varepsilon^{2} \nabla \frac{\partial U_{\varepsilon, y_{\varepsilon}}}{\partial x_{i}} \nabla \phi+V(x) \frac{\partial U_{\varepsilon, y_{\varepsilon}}}{\partial x_{i}} \phi\right)=0, \quad i=1, \cdots, N .
$$

We claim that the following estimates hold.

First, for any $R>0$,

$$
\int_{B_{\varepsilon R}\left(y_{\varepsilon}\right)} \omega_{\varepsilon}^{2}=o(1)\left\|\omega_{\varepsilon}\right\|_{\varepsilon}^{2}
$$

Second, for any given $R>0$, let $R_{\varepsilon}=\varepsilon^{\frac{2}{m+2}} R$. Then

$$
\varepsilon^{\frac{2 m}{m+2}} \int_{B_{R_{\varepsilon}}(0)} \omega_{\varepsilon}^{2}=o(1)\left\|\omega_{\varepsilon}\right\|_{\varepsilon}^{2}
$$

Finally,

$$
\varepsilon^{2} \int_{\Omega} \omega_{\varepsilon}^{2}=o(1)\left\|\omega_{\varepsilon}\right\|_{\varepsilon}^{2} .
$$

Suppose that (2.19), (2.20) and (2.21) are true. We will show from (2.19)-(2.21) that

$$
\int_{\mathbb{R}^{N}}\left(\Phi_{\varepsilon}+U_{\varepsilon, y_{\varepsilon}}\right)^{p-1} \omega_{\varepsilon}^{2}=o(1)\left\|\omega_{\varepsilon}\right\|_{\varepsilon}^{2}+o_{R}(1)\left\|\omega_{\varepsilon}\right\|_{\varepsilon}^{2},
$$

where $o_{R}(1)$ denotes quantities that go to 0 as $R \rightarrow \infty$.

To show (2.22), let us first give some estimates.

Similar to the proof of (2.14), we have

$$
\begin{aligned}
& \varepsilon^{\frac{2 m}{m+2}} \int_{B_{\delta}(0) \backslash B_{R_{\varepsilon}}(0)} \widehat{W}_{\varepsilon}^{p-1}\left(\varepsilon^{-\frac{2}{m+2}} x\right) \omega_{\varepsilon}^{2}(x) \\
& \leq o_{R}(1) \varepsilon^{\frac{2 m}{m+2}} \int_{B_{\delta}(0) \backslash B_{R_{\varepsilon}}(0)} \omega_{\varepsilon}^{2} \leq o_{R}(1)\left\|\omega_{\varepsilon}\right\|_{\varepsilon}^{2} .
\end{aligned}
$$


On the other hand, from $U_{\varepsilon, y_{\varepsilon}}(x)=o_{R}(1)$ for $x \in \mathbb{R}^{N} \backslash B_{\varepsilon R}\left(y_{\varepsilon}\right)$, we obtain

$$
\begin{aligned}
& \int_{B_{\delta}\left(y_{\varepsilon}\right) \backslash B_{\varepsilon R}\left(y_{\varepsilon}\right)} U_{\varepsilon}^{p-1}\left(\frac{x-y_{\varepsilon}}{\varepsilon}\right) \omega_{\varepsilon}^{2}(x) \\
& \leq o_{R}(1) \int_{B_{\delta}\left(y_{\varepsilon}\right) \backslash B_{\varepsilon R}\left(y_{\varepsilon}\right)} V(x) \omega_{\varepsilon}^{2} \leq o_{R}(1)\left\|\omega_{\varepsilon}\right\|_{\varepsilon}^{2} .
\end{aligned}
$$

By the exponential decay of $\widehat{W}_{\varepsilon}$ and $U$, we have

$$
\varepsilon^{\frac{2 m}{m+2}} \int_{\mathbb{R}^{N} \backslash B_{\delta}(0)} \widehat{W}_{\varepsilon}^{p-1}\left(\varepsilon^{-\frac{2}{m+2}} x\right) \omega_{\varepsilon}^{2}(x) \leq O\left(e^{-\gamma / \varepsilon}\right)\left\|\omega_{\varepsilon}\right\|_{\varepsilon}^{2}
$$

and

$$
\int_{\mathbb{R}^{N} \backslash B_{\delta}\left(y_{\varepsilon}\right)} U_{\varepsilon}^{p-1}\left(\frac{x-y_{\varepsilon}}{\varepsilon}\right) \omega_{\varepsilon}^{2} \leq O\left(e^{-\gamma / \varepsilon}\right)\left\|\omega_{\varepsilon}\right\|_{\varepsilon}^{2},
$$

where $\gamma>0$ is a small constant.

Combining all the above estimates, we obtain

$$
\begin{aligned}
& \int_{\mathbb{R}^{N}}\left(\Phi_{\varepsilon}+U_{\varepsilon, y_{\varepsilon}}\right)^{p-1} \omega_{\varepsilon}^{2} \\
\leq & C \int_{\mathbb{R}^{N}} W_{\varepsilon, y_{\varepsilon}}^{p-1} \omega_{\varepsilon}^{2}+C \int_{\mathbb{R}^{N}} \widetilde{W}_{\varepsilon}^{p-1} \omega_{\varepsilon}^{2}+C \int_{\mathbb{R}^{N}} U_{\varepsilon, y_{\varepsilon}}^{p-1} \omega_{\varepsilon}^{2}+O\left(e^{-\gamma / \varepsilon}\right)\left\|\omega_{\varepsilon}\right\|_{\varepsilon}^{2} \\
\leq & C \varepsilon^{2} \int_{\mathbb{R}^{N}}\left(\varepsilon^{-\frac{2}{p-1}} W_{\varepsilon}\right)^{p-1}|\omega|_{\varepsilon}^{2}+C \sup _{|x| \leq R_{\varepsilon}}\left(\varepsilon^{-\frac{2}{p-1} \frac{m}{m+2}} \widetilde{W}_{\varepsilon}(x)\right)^{p-1} \varepsilon^{\frac{2 m}{m+2}} \int_{B_{R_{\varepsilon}}(0)} \omega_{\varepsilon}^{2} \\
& +C \varepsilon^{\frac{2 m}{m+2}} \int_{B_{\delta}(0) \backslash B_{R_{\varepsilon}}(0)} \widehat{W}_{\varepsilon}^{p-1}\left(\varepsilon^{-\frac{2}{m+2}} x\right) \omega_{\varepsilon}^{2}+C \varepsilon^{\frac{2 m}{m+2}} \int_{\mathbb{R}^{N} \backslash B_{\delta}(0)} \widehat{W}_{\varepsilon}^{p-1}\left(\varepsilon^{-\frac{2}{m+2}} x\right) \omega_{\varepsilon}^{2} \\
& +C \int_{B_{\varepsilon R}\left(y_{\varepsilon}\right)} U^{p-1}\left(\frac{x-y_{\varepsilon}}{\varepsilon}\right) \omega_{\varepsilon}^{2}(x) d x \\
& +C \int_{\mathbb{R}^{N} \backslash B_{\delta}\left(y_{\varepsilon}\right)} U^{p-1}\left(\frac{x-y_{\varepsilon}}{\varepsilon}\right) \omega_{\varepsilon}^{2}(x) d x+C \int_{B_{\delta}\left(y_{\varepsilon}\right) \backslash B_{\varepsilon R}\left(y_{\varepsilon}\right)} U^{p-1}\left(\frac{x-y_{\varepsilon}}{\varepsilon}\right) \omega_{\varepsilon}^{2}(x) d x \\
\leq & C \varepsilon^{2} \int_{\Omega} \omega_{\varepsilon}^{2}(x) d x+o(1)\|\omega\|_{\varepsilon}^{2}+o_{R}(1)\|\omega\|_{\varepsilon}^{2} \\
= & o(1)\|\omega\|_{\varepsilon}^{2}+o_{R}(1)\|\omega\|_{\varepsilon}^{2},
\end{aligned}
$$

which implies (2.22).

Taking $\eta=\omega_{\varepsilon}$ in (2.17), we have from (2.22)

$$
\begin{aligned}
o(1)\left\|\omega_{\varepsilon}\right\|_{\varepsilon}^{2} & =\int_{\mathbb{R}^{N}}\left(\varepsilon^{2}\left|\nabla \omega_{\varepsilon}\right|^{2}+V(x) \omega_{\varepsilon}^{2}\right)-p \int_{\mathbb{R}^{N}}\left(\Phi_{\varepsilon}+U_{\varepsilon, y_{\varepsilon}}\right)^{p-1} \omega_{\varepsilon}^{2} \\
& =\left(1+o(1)+o_{R}(1)\right)\left\|\omega_{\varepsilon}\right\|_{\varepsilon}^{2},
\end{aligned}
$$

which is a contradiction.

It remains to prove (2.19), (2.20) and (2.21).

First, we prove (2.19). Let $\|\phi\|_{\varepsilon}^{*}=\left(\int_{\mathbb{R}^{N}}\left(|\nabla \phi|^{2}+V\left(\varepsilon x+y_{\varepsilon}\right) \phi^{2}\right) d x\right)^{\frac{1}{2}}$. Rescaling $\widetilde{\omega}_{\varepsilon}(x)=\omega_{\varepsilon}\left(\varepsilon x+y_{\varepsilon}\right)$, then by (2.17) we have

$\int_{\mathbb{R}^{N}}\left(\nabla \widetilde{\omega}_{\varepsilon} \nabla \eta+V\left(\varepsilon x+y_{\varepsilon}\right) \widetilde{\omega}_{\varepsilon} \eta\right)-p \int_{\mathbb{R}^{N}}\left(\Phi_{\varepsilon}\left(\varepsilon x+y_{\varepsilon}\right)+U(x)\right)^{p-1} \widetilde{\omega}_{\varepsilon} \eta=o(1)\left\|\widetilde{\omega}_{\varepsilon}\right\|_{\varepsilon}^{*}\|\eta\|_{\varepsilon}^{*}$ provided $\eta$ satisfies

$$
\int_{\mathbb{R}^{N}}\left(\nabla \frac{\partial U(x)}{\partial x_{i}} \nabla \eta+V\left(\varepsilon x+y_{\varepsilon}\right) \frac{\partial U(x)}{\partial x_{i}} \eta\right)=0, \quad i=1, \cdots, N .
$$


Without loss of generality, we assume $\left\|\widetilde{\omega}_{\varepsilon}\right\|_{\varepsilon}^{*}=1$. Since $V\left(y_{\varepsilon}\right) \geq c^{\prime \prime}>0$, we see that there is a $\widetilde{\omega} \in H^{1}\left(\mathbb{R}^{N}\right)$ such that

$$
\begin{array}{ll}
\widetilde{\omega}_{\varepsilon} \rightarrow \widetilde{\omega} & \text { weakly in } H_{\text {loc }}^{1}\left(\mathbb{R}^{N}\right), \\
\widetilde{\omega}_{\varepsilon} \rightarrow \widetilde{\omega} & \text { a.e. in } \mathbb{R}^{N} .
\end{array}
$$

We claim that $\widetilde{\omega}$ satisfies for all $\eta \in C_{0}^{\infty}\left(\mathbb{R}^{N}\right)$,

$$
\int_{\mathbb{R}^{N}}\left(\nabla \widetilde{\omega} \nabla \eta+V\left(a_{1}\right) \widetilde{\omega} \eta\right)=p \int_{\mathbb{R}^{N}} U^{p-1}(x) \widetilde{\omega} \eta
$$

In fact, for any given $\eta \in C_{0}^{\infty}\left(\mathbb{R}^{N}\right)$, by projecting $\eta$ into $\widetilde{E}_{\varepsilon, y_{\varepsilon}}$, the set of functions such that (2.24) is satisfied, we then have $c_{j, \varepsilon} \in \mathbb{R}(j=1, \cdots, N)$ such that $\eta_{\varepsilon}=$ $\eta-\sum_{j=1}^{N} c_{j, \varepsilon} \frac{\partial U(x)}{\partial x_{j}} \in \widetilde{E}_{\varepsilon, y_{\varepsilon}}$. It is not hard to show by (2.24) that $c_{j, \varepsilon}$ is uniformly bounded. Replacing $\eta$ in (2.23) by $\eta_{\varepsilon}$ we have

$$
\begin{gathered}
\int_{\mathbb{R}^{N}}\left(\nabla \widetilde{\omega}_{\varepsilon} \nabla\left(\eta-\sum_{j=1}^{N} c_{j, \varepsilon} \frac{\partial U(x)}{\partial x_{j}}\right)+V\left(\varepsilon x+y_{\varepsilon}\right) \widetilde{\omega}_{\varepsilon}\left(\eta-\sum_{j=1}^{N} c_{j, \varepsilon} \frac{\partial U(x)}{\partial x_{j}}\right)\right) \\
-p \int_{\mathbb{R}^{N}}\left(\Phi_{\varepsilon}\left(\varepsilon x+y_{\varepsilon}\right)+U(x)\right)^{p-1} \widetilde{\omega}_{\varepsilon}\left(\eta-\sum_{j=1}^{N} c_{j, \varepsilon} \frac{\partial U(x)}{\partial x_{j}}\right) \\
=o(1)\left\|\widetilde{\omega}_{\varepsilon}\right\|_{\varepsilon}^{*}\|\eta\|_{\varepsilon}^{*} .
\end{gathered}
$$

Taking $\varepsilon \rightarrow 0$ in (2.26), we have from $y_{\varepsilon} \rightarrow a_{1}$,

$$
\begin{aligned}
& \int_{\mathbb{R}^{N}}\left(\nabla \widetilde{\omega} \nabla \eta+V\left(a_{1}\right) \widetilde{\omega} \eta\right)-p \int_{\mathbb{R}^{N}} U^{p-1}(x) \widetilde{\omega} \eta \\
& -\sum_{j=1}^{N} c_{j}\left(\int_{\mathbb{R}^{N}}\left(\nabla \widetilde{\omega} \nabla \frac{\partial U(x)}{\partial x_{j}}+V\left(a_{1}\right) \widetilde{\omega} \frac{\partial U(x)}{\partial x_{j}}\right)-p \int_{\mathbb{R}^{N}} U^{p-1}(x) \widetilde{\omega} \frac{\partial U(x)}{\partial x_{j}}\right)=0
\end{aligned}
$$

where $c_{j, \varepsilon} \rightarrow c_{j}$ (up to a subsequence). But

$$
\int_{\mathbb{R}^{N}}\left(\nabla \widetilde{\omega} \nabla \frac{\partial U(x)}{\partial x_{j}}+V\left(a_{1}\right) \widetilde{\omega} \frac{\partial U(x)}{\partial x_{j}}\right)-p \int_{\mathbb{R}^{N}} U^{p-1}(x) \widetilde{\omega} \frac{\partial U(x)}{\partial x_{j}}=0 .
$$

Thus, (2.25) follows.

On the other hand, by $\widetilde{\omega}_{\varepsilon} \in \widetilde{E}_{\varepsilon, y_{\varepsilon}}$ we have

$$
\int_{\mathbb{R}^{N}}\left(\nabla \widetilde{\omega} \nabla \frac{\partial U(x)}{\partial x_{j}}+V\left(a_{1}\right) \widetilde{\omega} \frac{\partial U(x)}{\partial x_{j}}\right)=0, \quad i=1, \cdots, N .
$$

From the fact that $U$ is non-degenerate, we have $\widetilde{\omega} \equiv 0$ in $\mathbb{R}^{N}$ (see [18, for example). Therefore for any $R>0$,

$$
\int_{B_{R}(0)} \widetilde{\omega}_{\varepsilon}^{2} \rightarrow 0 \quad \text { as } \varepsilon \rightarrow 0
$$

which, together with $\left\|\omega_{\varepsilon}\right\|_{\varepsilon}^{2}=\varepsilon^{N}\left(\left\|\widetilde{\omega}_{\varepsilon}\right\|_{\varepsilon}^{*}\right)^{2}=\varepsilon^{N}$, yields (2.19).

Now we turn to the proof of (2.20).

Set $\widehat{\omega}(x)=\varepsilon^{-\frac{2 m}{(p-1)(m+2)} \omega}\left(\varepsilon^{\frac{2}{m+2}} x\right)$. Define

$$
|[\widehat{\omega}]|_{\varepsilon}=\left(\int_{\mathbb{R}^{N}}\left(|\nabla \widehat{\omega}|^{2}+\varepsilon^{-\frac{2 m}{m+2}} V\left(\varepsilon^{\frac{2}{m+2}} x\right) \widehat{\omega}^{2}(x)\right) d x\right)^{\frac{1}{2}} .
$$


Assume $\left|\left[\widehat{\omega}_{\varepsilon}\right]\right|_{\varepsilon}=1$ (otherwise take $\frac{\widehat{\omega}_{\varepsilon}}{\left.\left[\hat{\omega}_{\varepsilon}\right]\right|_{\varepsilon}}$ ). Then $(2.17)$ is equivalent to

$$
\begin{aligned}
& \int_{\mathbb{R}^{N}}\left(\nabla \widehat{\omega}_{\varepsilon} \nabla \widehat{\eta}+\varepsilon^{-\frac{2 m}{m+2}} V\left(\varepsilon^{\frac{2}{m+2}} x\right) \widehat{\omega}_{\varepsilon} \widehat{\eta}\right) \\
& -p \varepsilon^{-\frac{2 m}{m+2}} \int_{\mathbb{R}^{N}}\left(\Phi_{\varepsilon}\left(\varepsilon^{\frac{2}{m+2}} x\right)+U\left(\frac{\varepsilon^{\frac{2}{m+2}} x-y_{\varepsilon}}{\varepsilon}\right)\right)^{p-1} \widehat{\omega}_{\varepsilon} \widehat{\eta} \\
= & o(1)\left|\left[\widehat{\omega}_{\varepsilon}\right]\right|_{\varepsilon}|[\widehat{\eta}]|_{\varepsilon},
\end{aligned}
$$

for any $\widehat{\eta} \in H^{1}\left(\mathbb{R}^{N}\right)$, satisfying

$$
\int_{\mathbb{R}^{N}}\left(\nabla \widehat{U}_{\varepsilon, j} \nabla \widehat{\eta}+\varepsilon^{-\frac{2 m}{m+2}} V\left(\varepsilon^{\frac{2}{m+2}} x\right) \widehat{U}_{\varepsilon, j} \widehat{\eta}\right)=0
$$

where $\widehat{U}_{\varepsilon, j}(x)=\left.\varepsilon^{-\frac{2 m}{(p-1)(m+2)}} \frac{\partial U_{\varepsilon, y_{\varepsilon}}(z)}{\partial x_{j}}\right|_{z=\varepsilon^{\frac{2}{m+2}} x}$.

Since $\varepsilon^{-\frac{2 m}{m+2}} V\left(\varepsilon^{\frac{2}{m+2}} x\right) \geq c^{\prime}|x|^{m}$ if $|x| \leq R$, it is not difficult to prove that there is an $\widehat{\omega} \in H^{1}\left(\mathbb{R}^{N}\right)$, such that

$$
\begin{gathered}
\widehat{\omega}_{\varepsilon} \rightarrow \widehat{\omega} \quad \text { weakly in } H_{\mathrm{loc}}^{1}\left(\mathbb{R}^{N}\right), \\
\widehat{\omega}_{\varepsilon} \rightarrow \widehat{\omega} \quad \text { a.e. in } \mathbb{R}^{N} .
\end{gathered}
$$

For any $\eta \in C_{0}^{\infty}\left(\mathbb{R}^{N}\right)$, choose $f_{\varepsilon, j}$, such that $\eta_{\varepsilon}=\eta-\sum_{j=1}^{N} f_{\varepsilon, j} \widehat{U}_{\varepsilon, j}$ satisfies (2.29). Since $\eta$ has compact support, and $\widehat{U}_{\varepsilon, j}$ is exponentially small in $B_{R}(0)$ for any $R>0$, we see that $\left|f_{\varepsilon, j}\right| \leq e^{-\gamma / \varepsilon}$ for some $\gamma>0$.

Inserting $\eta_{\varepsilon}$ into (2.28), using

$$
\begin{aligned}
& \varepsilon^{-\frac{2 m}{m+2}} \int_{\mathbb{R}^{N}}\left(\Phi_{\varepsilon}\left(\varepsilon^{\frac{2}{m+2}} x\right)+U\left(\frac{\varepsilon^{\frac{2}{m+2}} x-y_{\varepsilon}}{\varepsilon}\right)\right)^{p-1} \widehat{\omega}_{\varepsilon} \eta \\
& =\int_{\mathbb{R}^{N}}\left(\varepsilon^{-\frac{2 m}{(p-1)(m+2)}} W_{\varepsilon}\left(\varepsilon^{\frac{2}{m+2}} x\right)+\varepsilon^{-\frac{2 m}{(p-1)(m+2)}} \widetilde{W}_{\varepsilon}\left(\varepsilon^{\frac{2}{m+2}} x\right)+U\left(\frac{\varepsilon^{\frac{2}{m+2}} x-y_{\varepsilon}}{\varepsilon}\right)\right)^{p-1} \widehat{\omega}_{\varepsilon} \eta \\
& \longrightarrow \int_{\mathbb{R}^{N}} \widehat{W}^{p-1}(x) \widehat{\omega} \eta,
\end{aligned}
$$

and

$$
\int_{\mathbb{R}^{N}} \varepsilon^{-\frac{2 m}{m+2}} V\left(\varepsilon^{\frac{2}{m+2}} x\right) \widehat{\omega} \eta \longrightarrow \int_{\mathbb{R}^{N}}|x|^{m} \widehat{\omega} \eta,
$$

we obtain that for any $\eta \in C_{0}^{\infty}\left(\mathbb{R}^{N}\right)$,

$$
\int_{\mathbb{R}^{N}}\left(\nabla \widehat{\omega} \nabla \eta+|x|^{m} \widehat{\omega} \eta\right)=p \int_{\mathbb{R}^{N}} \widehat{W}^{p-1} \widehat{\omega} \eta,
$$

which, by the fact that $\widehat{W}$ is non-degenerate, implies that $\widehat{\omega} \equiv 0$. Thus for any given $R>0$,

$$
\int_{B_{R}(0)} \widehat{\omega}_{\varepsilon}^{2} \rightarrow 0
$$

which gives

$$
\int_{B_{R_{\varepsilon}}(0)} \omega_{\varepsilon}^{2}=o(1) \varepsilon^{\frac{2 N}{m+2}+\frac{4 m}{(p-1)(m+2)}} .
$$

From $|[\widehat{\omega}]|_{\varepsilon}^{2}=1$, we deduce

$$
\left\|\omega_{\varepsilon}\right\|_{\varepsilon}^{2}=\varepsilon^{\frac{2 N}{m+2}+\frac{4 m}{(p-1)(m+2)}+\frac{2 m}{m+2}} .
$$


Thus,

$$
\varepsilon^{\frac{2 m}{m+2}} \int_{B_{R_{\varepsilon}}(0)} \omega_{\varepsilon}^{2}=o(1) \varepsilon^{\frac{2 N}{m+2}+\frac{4 m}{(p-1)(m+2)}+\frac{2 m}{m+2}}=o(1)\left\|\omega_{\varepsilon}\right\|_{\varepsilon}^{2},
$$

and (2.20) follows.

To show (2.21), we define

$$
|[\omega]|_{\varepsilon}^{*}=\left(\int_{\mathbb{R}^{N}}\left(|\nabla \omega|^{2}+\varepsilon^{-2} V(x) \omega^{2}(x)\right) d x\right)^{\frac{1}{2}} .
$$

Then, (2.17) is equivalent to

$$
\int_{\mathbb{R}^{N}} \nabla \omega_{\varepsilon} \nabla \eta+\varepsilon^{-2} V(x) \omega_{\varepsilon} \eta-p \varepsilon^{-2} \int_{\mathbb{R}^{N}}\left(\Phi_{\varepsilon}+U_{\varepsilon, y}\right)^{p-1} \omega_{\varepsilon} \eta=o(1)\left|\left[\omega_{\varepsilon}\right]\right|_{\varepsilon}^{*}|[\eta]|_{\varepsilon}^{*},
$$

for any $\eta \in E_{\varepsilon, y_{\varepsilon}}$.

Assume $\left|\left[\omega_{\varepsilon}\right]\right|_{\varepsilon}^{*}=1$ (otherwise replace $\omega_{\varepsilon}$ by $\frac{\omega_{\varepsilon}}{\left[\left(\omega_{\varepsilon}\right]\right]_{\varepsilon}^{*}}$ ). Then $\omega_{\varepsilon}$ is bounded in $H^{1}\left(\mathbb{R}^{N}\right)$. Consequently there exists an $\omega \in H^{1}\left(\mathbb{R}^{N}\right)$ such that

$$
\begin{gathered}
\omega_{\varepsilon} \rightarrow \omega \quad \text { weakly in } H^{1}\left(\mathbb{R}^{N}\right), \\
\omega_{\varepsilon} \rightarrow \omega \quad \text { a.e. in } \mathbb{R}^{N} .
\end{gathered}
$$

Moreover from $\int_{\mathbb{R}^{N}} V(x) \omega_{\varepsilon}^{2} \leq \varepsilon^{2}$, we obtain $\omega=0$ for $x \in \mathbb{R}^{N} \backslash \mathcal{Z}$. For any $\eta \in$ $H_{0}^{1}(\Omega)$, let $\eta_{\varepsilon}=\eta-\sum_{j=1}^{N} d_{j, \varepsilon} \frac{\partial U_{\varepsilon, y_{\varepsilon}}(x)}{\partial x_{j}}$, where $d_{j, \varepsilon} \in \mathbb{R}$ is chosen so that $\eta_{\varepsilon} \in E_{\varepsilon, y_{\varepsilon}}$. It is not difficult to check that $d_{j, \varepsilon}=O\left(e^{-\gamma / \varepsilon}\right)$ for some $\gamma>0$ from the fact that $U_{\varepsilon, y_{\varepsilon}}(x) \leq C e^{-\sqrt{V\left(x_{0}\right)}\left|x-y_{\varepsilon}\right| / \varepsilon}$ and $y_{\varepsilon} \rightarrow x_{0}$.

Taking $\eta=\eta_{\varepsilon}$ in (2.30) and letting $\varepsilon \rightarrow 0$ we get

$$
\int_{\Omega} \nabla \omega \nabla \eta=p \int_{\Omega} W^{p-1} \omega \eta
$$

since both $W_{\varepsilon}(x)$ and $U_{\varepsilon, y_{\varepsilon}}$ are bounded by $e^{-\gamma / \varepsilon}$ for $x \in \Omega$, where $\gamma>0$ is a constant. Therefore $\omega \equiv 0$ in $\mathbb{R}^{N}$. Thus

$$
\int_{\Omega} \omega_{\varepsilon}^{2}=o(1)
$$

which, in view of $\|\omega\|_{\varepsilon}^{2}=\varepsilon^{2}\left(|[\omega]|_{\varepsilon}^{*}\right)^{2}=\varepsilon^{2}$, implies (2.21). We therefore complete our proof of Proposition 2.3

Denote $\widehat{\Omega}=\Omega \cup\{0\}$ and define for $\omega, \eta \in H_{0}^{1}\left(\widehat{\Omega}^{2 \delta}\right)$ the inner product of $\omega$ and $\eta$ :

$$
\begin{gathered}
(\omega, \eta)_{\varepsilon}=\int_{\widehat{\Omega}^{2 \delta}}\left(\nabla \omega \nabla \eta+\varepsilon^{-2} V(x) \omega \eta\right) d x, \\
\widetilde{L}_{\varepsilon} \omega=-\Delta \omega+\varepsilon^{-2} V(x) \omega-\varepsilon^{-2} p\left(\Phi_{\varepsilon}(x)+U_{\varepsilon, y}(x)\right)^{p-1} \omega .
\end{gathered}
$$

Proposition 2.4. Suppose (V2-2) and (V3-2) hold. Then there exists $\rho_{1}>0$ independent of $\varepsilon$ such that

$$
\left|\left(\widetilde{L}_{\varepsilon} \omega\right)\right|_{\varepsilon} \geq \rho_{1}|(\omega)|_{\varepsilon} \text { for any } \omega \in H_{0}^{1}\left(\widehat{\Omega}^{2 \delta}\right)
$$

where $\mid(\cdot)_{\varepsilon}$ is the norm defined by the inner product $(\cdot, \cdot)_{\varepsilon}$. 
Proposition 2.4 can be proved by the similar arguments to those used in the proof of Proposition 2.3 and therefore we omit it here.

Define

$$
\begin{aligned}
\mathcal{S}_{\varepsilon}=\left\{\omega \mid \omega \in E_{\varepsilon, y},\right. & \|\omega\|_{\varepsilon} \leq \varepsilon^{\frac{N}{2}+2-\tau},|\omega(x)| \leq \varepsilon^{\sigma} e^{-\sigma|x-y| /(\varepsilon \delta)} \quad \text { for } \quad x \in B_{\delta}(y), \\
& \left.|\omega(x)| \leq e^{-\sigma / \varepsilon} \text { for } \quad x \in \mathbb{R}^{N} \backslash B_{\delta}(y)\right\}
\end{aligned}
$$

where $\sigma>0$ and $\tau \in(0, \sigma)$ are small constants to be determined.

Proposition 2.5. There exists $\varepsilon_{2}>0$ such that for $\varepsilon \in\left(0, \varepsilon_{2}\right)$ there is a unique $C^{1}$ map: $y \in B_{\varepsilon}\left(a_{1}\right) \rightarrow \omega_{\varepsilon} \in H^{1}\left(\mathbb{R}^{N}\right)$ satisfying $\omega_{\varepsilon} \in \mathcal{S}_{\varepsilon}$ and for some $D_{j, \varepsilon} \in \mathbb{R}$

$$
I^{\prime}\left(U_{\varepsilon, y}+\omega_{\varepsilon}\right)=\sum_{j=1}^{N} D_{j, \varepsilon} \frac{\partial U_{\varepsilon, y}}{\partial x_{j}} .
$$

Moreover

$$
\left\|\omega_{\varepsilon}\right\|_{\varepsilon}=O\left(\varepsilon^{\frac{N}{2}+2}\right)
$$

and

$$
D_{j, \varepsilon}=O\left(\varepsilon^{3}\right) .
$$

Proof. By Lemma 2.1, there is $\omega_{\ell_{\varepsilon}} \in E_{\varepsilon, y}$ such that

$$
\left\langle\omega_{l_{\varepsilon}}, \omega\right\rangle_{\varepsilon}=\ell_{\varepsilon}(\omega) \quad \text { for any } \omega \in E_{\varepsilon, y}
$$

and

$$
\begin{aligned}
& \left\|\omega_{\ell_{\varepsilon}}\right\|_{\varepsilon} \leq C\left(\left|V(y)-V\left(a_{1}\right)\right|+\varepsilon|\nabla V(y)|+\varepsilon^{2}\right) \varepsilon^{\frac{N}{2}} \\
= & O\left(\left|y-a_{1}\right|^{2}+\varepsilon\left|y-a_{1}\right|+\varepsilon^{2}\right) \varepsilon^{\frac{N}{2}}=O\left(\varepsilon^{\frac{N}{2}+2}\right) .
\end{aligned}
$$

It follows from Lemma 2.2 and Proposition 2.3 that there exists an invertible linear operator $L_{\varepsilon}$ such that

$$
Q_{\varepsilon}(\omega, \omega)=\left\langle L_{\varepsilon} \omega, \omega\right\rangle_{\varepsilon} \quad \text { for all } \omega \in E_{\varepsilon, y} .
$$

Hence to find a critical point of $I$ is equivalent to solving

$$
\omega_{\ell_{\varepsilon}}+L_{\varepsilon} \omega+R_{\varepsilon}^{\prime}(\omega)=0 .
$$

Define $G_{\varepsilon}(\omega)=-L_{\varepsilon}^{-1} \omega_{\ell_{\varepsilon}}-L_{\varepsilon}^{-1} R_{\varepsilon}^{\prime}(\omega)$; then to solve (2.34) is equivalent to finding a fixed point of $G_{\varepsilon}$ in $E_{\varepsilon, y}$. We will establish the existence of a fixed point $\omega_{\varepsilon, y}$ for $y \in B_{\varepsilon}\left(a_{1}\right)$ by the following four steps.

Step 1. There are $C>0, \vartheta \in(0,1)$ such that

$$
\begin{aligned}
\left\|G_{\varepsilon}(\omega)\right\|_{\varepsilon} \leq C \varepsilon^{\frac{N}{2}+2} \leq \varepsilon^{\frac{N}{2}+2-\tau} & \text { for any } \omega \in S_{\varepsilon}, \\
\left\|G_{\varepsilon}\left(\omega_{1}\right)-G_{\varepsilon}\left(\omega_{2}\right)\right\|_{\varepsilon} \leq \vartheta\left\|\omega_{1}-\omega_{2}\right\|_{\varepsilon} & \text { for any } \omega_{1}, \omega_{2} \in S_{\varepsilon} .
\end{aligned}
$$

By direct calculations, we have

$$
\left\langle R_{\varepsilon}^{\prime}(\omega), \eta\right\rangle=\int_{\mathbb{R}^{N}}\left[\left(\Phi_{\varepsilon}+U_{\varepsilon, y}+\omega\right)_{+}^{p}-\left(\Phi_{\varepsilon}+U_{\varepsilon, y}\right)^{p}-p\left(\Phi_{\varepsilon}+U_{\varepsilon, y}\right)^{p-1} \omega\right] \eta .
$$


Let $\bar{p}=\min \{p, 2\}$. Then (2.37)

$$
\begin{aligned}
\left|\left\langle R_{\varepsilon}^{\prime}(\omega), \eta\right\rangle\right| & \leq C \int_{\mathbb{R}^{N}}\left(|\omega|^{p}+|\omega|^{\bar{p}}\right)|\eta| \\
& =C\left(\int_{B_{\delta}(y)}\left(|\omega|^{p}+|\omega|^{\bar{p}}\right)|\eta|+\int_{\mathbb{R}^{N} \backslash B_{\delta}(y)}\left(|\omega|^{p}+|\omega|^{\bar{p}}\right)|\eta|\right) \\
& \leq C \varepsilon^{\sigma(\bar{p}-1)} \int_{B_{\delta}(y)} V(x)|\omega||\eta|+C e^{-\sigma(\bar{p}-1) / \varepsilon} \int_{\mathbb{R}^{N} \backslash B_{\delta}(y)}|\omega||\eta| \\
& \leq C \varepsilon^{\sigma(\bar{p}-1)}\|\omega\|_{\varepsilon}\|\eta\|_{\varepsilon} \leq C \varepsilon^{\sigma(\bar{p}-1)+\frac{N}{2}+2-\tau}\|\eta\|_{\varepsilon} \\
& \leq \varepsilon^{\frac{N}{2}+2}\|\eta\|_{\varepsilon},
\end{aligned}
$$

provided $\tau<(\bar{p}-1) \sigma$, and $\varepsilon_{2}$ is small enough. Thus,

$$
\left\|G_{\varepsilon}(\omega)\right\|_{\varepsilon} \leq C\left\|\omega_{\ell_{\varepsilon}}\right\|_{\varepsilon}+C\left\|R_{\varepsilon}^{\prime}(\omega)\right\|_{\varepsilon} \leq C \varepsilon^{\frac{N}{2}+2} .
$$

So (2.35) is proved.

By direct calculation, we have

$$
\begin{aligned}
& \left\langle R_{\varepsilon}^{\prime}\left(\omega_{1}\right)-R_{\varepsilon}^{\prime}\left(\omega_{2}\right), \eta\right\rangle \\
= & \int_{\mathbb{R}^{N}}\left[\left(\Phi_{\varepsilon}+U_{\varepsilon, y}+\omega_{1}\right)_{+}^{p}-\left(\Phi_{\varepsilon}+U_{\varepsilon, y}+\omega_{2}\right)_{+}^{p}-p\left(\Phi_{\varepsilon}+U_{\varepsilon, y}\right)^{p-1}\left(\omega_{1}-\omega_{2}\right)\right] \eta,
\end{aligned}
$$

from which we deduce by using argument as in (2.37),

$$
\begin{aligned}
& \left|\left\langle R_{\varepsilon}^{\prime}\left(\omega_{1}\right)-R_{\varepsilon}^{\prime}\left(\omega_{2}\right), \eta\right\rangle\right| \\
\leq & C \int_{\mathbb{R}^{N}}\left(\left|\omega_{1}\right|^{p-1}+\left|\omega_{2}\right|^{p-1}+\left(\Phi_{\varepsilon}+U_{\varepsilon, y}\right)^{p-2}\left(\left|\omega_{1}\right|+\left|\omega_{2}\right|\right)\right)\left|\omega_{1}-\omega_{2}\right||\eta| \\
\leq & C \varepsilon^{\frac{\sigma}{4}(\bar{p}-1)}\left\|\omega_{1}-\omega_{2}\right\|_{\varepsilon}\|\eta\|_{\varepsilon} .
\end{aligned}
$$

So, (2.36) follows directly from (2.38).

Step 2. Let $\omega_{1}=G_{\varepsilon}(\omega)$. Then there is a small $\mu>0$, such that

$$
\left|\omega_{1}(x)\right| \leq C \varepsilon^{(1+\mu) \sigma} \quad \text { for all } \quad x \in \mathbb{R}^{N} \backslash \mathcal{Z}^{\delta} .
$$

Indeed, $\omega_{1}$ satisfies $L_{\varepsilon} \omega_{1}=-\omega_{\ell_{\varepsilon}}-R_{\varepsilon}^{\prime}(\omega)$ and there are $D_{j, \varepsilon} \in \mathbb{R}$ for $j=1, \cdots, N$ such that for any $\eta \in H^{1}\left(\mathbb{R}^{N}\right)$

$$
\left\langle L_{\varepsilon} \omega_{1}, \eta\right\rangle_{\varepsilon}=-\left\langle\omega_{\ell_{\varepsilon}}, \eta\right\rangle_{\varepsilon}-\left\langle R_{\varepsilon}^{\prime}(\omega), \eta\right\rangle+\sum_{j=1}^{N} D_{j, \varepsilon}\left\langle\frac{\partial U_{\varepsilon, y}}{\partial x_{j}}, \eta\right\rangle_{\varepsilon} .
$$

We claim that $D_{j, \varepsilon}=O\left(\varepsilon^{3}\right)$ for $j=1, \cdots, N$. To this end, taking $\eta=\frac{\partial U_{\varepsilon, y}}{\partial x_{j}}$ respectively in (2.40) we obtain a linear system for $D_{j, \varepsilon}$ $(2.41)$

$$
\sum_{j=1}^{N}\left\langle\frac{\partial U_{\varepsilon, y}}{\partial x_{i}}, \frac{\partial U_{\varepsilon, y}}{\partial x_{j}}\right\rangle_{\varepsilon} D_{j, \varepsilon}=\left\langle L_{\varepsilon} \omega_{1}, \frac{\partial U_{\varepsilon, y}}{\partial x_{j}}\right\rangle_{\varepsilon}+\left\langle\omega_{\ell_{\varepsilon}}, \frac{\partial U_{\varepsilon, y}}{\partial x_{j}}\right\rangle_{\varepsilon}+\left\langle R_{\varepsilon}^{\prime}(\omega), \frac{\partial U_{\varepsilon, y}}{\partial x_{j}}\right\rangle .
$$


With direct calculations, we have

$$
\begin{gathered}
\left|\left\langle L_{\varepsilon} \omega_{1}, \frac{\partial U_{\varepsilon, y}}{\partial x_{j}}\right\rangle_{\varepsilon}\right| \leq C\left\|\omega_{1}\right\|_{\varepsilon}\left\|\frac{\partial U_{\varepsilon, y}}{\partial x_{j}}\right\|_{\varepsilon} \leq C \varepsilon^{N+1}, \\
\left|\left\langle\omega_{\ell_{\varepsilon}}, \frac{\partial U_{\varepsilon, y}}{\partial x_{j}}\right\rangle_{\varepsilon}\right| \leq C\left\|\omega_{\ell_{\varepsilon}}\right\|_{\varepsilon}\left\|\frac{\partial U_{\varepsilon, y}}{\partial x_{j}}\right\|_{\varepsilon} \leq C \varepsilon^{N+1}, \\
\left|\left\langle R_{\varepsilon}^{\prime}(\omega), \frac{\partial U_{\varepsilon, y}}{\partial x_{j}}\right\rangle\right| \leq C\left\|R_{\varepsilon}^{\prime}(\omega)\right\|_{\varepsilon}\left\|\frac{\partial U_{\varepsilon, y}}{\partial x_{j}}\right\|_{\varepsilon} \leq C \varepsilon^{N+1},
\end{gathered}
$$

and

$$
\left\langle\frac{\partial U_{\varepsilon, y}}{\partial x_{i}}, \frac{\partial U_{\varepsilon, y}}{\partial x_{j}}\right\rangle_{\varepsilon}= \begin{cases}c_{0} \varepsilon^{N-2}, & \text { if } i=j, i, j=1, \cdots, N \\ O\left(\varepsilon^{N}\right), & \text { if } i \neq j, i, j=1, \cdots, N\end{cases}
$$

for some positive constant $c_{0}$, depending on $V\left(a_{1}\right), p$, and $N$ only. We obtain our conclusion easily from the estimates in (2.41).

To obtain an $L^{\infty}$ estimate for $\omega_{1}$ in $\mathbb{R}^{N} \backslash \mathcal{Z}^{\delta}$ we need to make use of the following equation satisfied by $\omega_{1}$ :

$$
-\varepsilon^{2} \Delta \omega_{1}+V(x) \omega_{1}-p\left(\Phi_{\varepsilon}(x)+U_{\varepsilon, y}\right)^{p-1} \omega_{1}=f_{\varepsilon}(x)
$$

with $f_{\varepsilon}(x)=f_{\varepsilon}^{1}(x)+f_{\varepsilon}^{2}(x)+f_{\varepsilon}^{3}(x)$, where $f_{\varepsilon}^{i}(i=1,2,3)$ is given by

$$
\begin{gathered}
f_{\varepsilon}^{1}(x)=\left(V\left(a_{1}\right)-V(x)\right) U_{\varepsilon, y}(x)-\left[\left(\Phi_{\varepsilon}+U_{\varepsilon, y}\right)^{p}-W_{\varepsilon}^{p}-\widehat{W}_{\varepsilon}^{p}-U_{\varepsilon, y}^{p}\right], \\
f_{\varepsilon}^{2}(x)=-\left[\left(\Phi_{\varepsilon}+U_{\varepsilon, y}+\omega\right)_{+}^{p}-\left(\Phi_{\varepsilon}+U_{\varepsilon, y}\right)^{p}-p\left(\Phi_{\varepsilon}+U_{\varepsilon, y}\right)^{p-1} \omega\right],
\end{gathered}
$$

and

$$
f_{\varepsilon}^{3}(x)=\sum_{j=1}^{N}\left[\left(V(x)-V\left(a_{1}\right)\right) \frac{\partial U_{\varepsilon, y}(x)}{\partial x_{j}}+p U_{\varepsilon, y}^{p-1} \frac{\partial U_{\varepsilon, y}(x)}{\partial x_{j}}\right] D_{j, \varepsilon} .
$$

By the definition, we can find a constant $\gamma>0$, such that (2.46)

$$
\begin{aligned}
\left|f_{\varepsilon}^{1}(x)\right| \leq & \left|V\left(x_{0}\right)-V(y)\right| U_{\varepsilon, y}(x)+|V(x)-V(y)| U_{\varepsilon, y}(x) \\
& +C\left[W_{\varepsilon}^{p-1}\left(W_{\varepsilon}+U_{\varepsilon, y}\right)+\widehat{W}_{\varepsilon}^{p-1}\left(W_{\varepsilon}+U_{\varepsilon, y}\right)+U_{\varepsilon, y}^{p-1}\left(W_{\varepsilon}+\widehat{W}_{\varepsilon}\right)\right] \\
& \leq\left|V\left(x_{0}\right)-V(y)\right| U_{\varepsilon, y}(x)+C|x-y| U_{\varepsilon, y}(x)+O\left(e^{-\gamma / \varepsilon}\right) \\
& \leq C \varepsilon,
\end{aligned}
$$

and

$$
\left|f_{\varepsilon}^{2}(x)\right| \leq C\left(|\omega|^{p}+|\omega|^{\bar{p}}\right) \leq C \varepsilon^{\sigma \bar{p}} \leq \varepsilon^{(1+\mu) \sigma} .
$$

Since $D_{j, \varepsilon}=O\left(\varepsilon^{3}\right)$, we have

$$
\left|f_{\varepsilon}^{3}(x)\right| \leq \varepsilon
$$

Set $\widetilde{\omega}_{1}(x)=\omega_{1}(\varepsilon x)$; then

$$
-\Delta \widetilde{\omega}_{1}+V(\varepsilon x) \widetilde{\omega}_{1}=f_{\varepsilon}(\varepsilon x)+p\left(\Phi_{\varepsilon}(\varepsilon x)+U\left(x-\varepsilon^{-1} y\right)\right)^{p-1} \widetilde{\omega}_{1} .
$$

From $\left\|\omega_{1}\right\|_{\varepsilon} \leq C \varepsilon^{\frac{N}{2}+2}$, we have for $z \in \mathbb{R}^{N} \backslash \mathcal{Z}_{\varepsilon}^{\frac{\delta}{3}}$

$$
\left(\int_{B_{2}(z)} \widetilde{\omega}_{1}^{2}\right)^{\frac{1}{2}} \leq C \varepsilon^{2}
$$


By the Moser iteration technique (see Gilbarg and Trudinger [19]), using the fact that $\left(\Phi_{\varepsilon}(\varepsilon x)+U\left(x-\varepsilon^{-1} y\right)\right)^{p-1}$ is bounded, we have

$$
\begin{aligned}
\left\|\widetilde{\omega}_{1}\right\|_{L^{\infty}\left(B_{1}(z)\right)} & \leq C\left(\left\|\widetilde{\omega}_{1}\right\|_{L^{2}\left(B_{2}(z)\right)}+\left\|f_{\varepsilon}\right\|_{L^{\infty}\left(B_{2}(z)\right)}\right) \\
& \leq C \varepsilon^{(1+\mu) \sigma},
\end{aligned}
$$

which implies (2.39). So, we complete step 2.

Step 3. There exists $\mu>0$ such that

$$
\left|\omega_{1}(x)\right| \leq e^{-(1+\mu) \sigma / \varepsilon} \text { for } x \in \mathbb{R}^{N} \backslash B_{\delta}(y) .
$$

Fix $\theta \in(0,1)$. By (2.43), we have from (1.9) and (1.10)

$$
\left|f_{\varepsilon}^{1}(x)\right| \leq e^{-(1+10 \mu) \sigma / \varepsilon} \text { for } x \in \mathbb{R}^{N} \backslash B_{\delta(1-\theta)}(y),
$$

provided $\sigma$ is small such that $(1+10 \mu) \sigma<\sqrt{V\left(x_{0}\right)} \delta(1-\theta)$, and

$$
\begin{aligned}
& \left|f_{\varepsilon}^{2}(x)\right| \leq C\left(|\omega|^{p}+|\omega|^{\bar{p}}\right) \\
& \leq \begin{cases}C e^{-\bar{p} \sigma / \varepsilon}, & x \in \mathbb{R}^{N} \backslash B_{\delta}(y), \\
C e^{-\bar{p} \sigma(1-\theta) / \varepsilon}, & x \in B_{\delta}(y) \backslash B_{\delta(1-\theta)}(y)\end{cases} \\
& \leq e^{-(1+10 \mu) \sigma / \varepsilon}, \quad x \in \mathbb{R}^{N} \backslash B_{\delta(1-\theta)}(y),
\end{aligned}
$$

provided $\mu$ is chosen so that $\bar{p}(1-\theta)>1+10 \mu$.

Similarly

$$
\left|f_{\varepsilon}^{3}(x)\right| \leq e^{-(1+10 \mu) \sigma / \varepsilon}, \quad x \in \mathbb{R}^{N} \backslash B_{\delta(1-\theta)}(y) .
$$

Suppose $\delta>0, \gamma_{0}>0$ are small so that $V(x) \geq 6 \gamma_{0}$ for $x \in \mathbb{R}^{N} \backslash \mathcal{Z}^{\delta}$. Let $\Psi_{1}$ be the solution of

$$
\left\{\begin{array}{l}
-\varepsilon^{2} \Delta \Psi+4 \gamma_{0} \Psi=0, \quad x \in \mathbb{R}^{N} \backslash \mathcal{Z}^{\delta}, \\
\Psi(x) \rightarrow 0 \text { as }|x| \rightarrow \infty, \\
\Psi=1 \quad \text { for } x \in \partial \mathcal{Z}^{\delta}
\end{array}\right.
$$

By maximum principle, it is not difficult to see that

$$
0<\Psi_{1}(x) \leq C e^{-3 \gamma_{0} \operatorname{dist}\left(x, \mathcal{Z}^{\delta}\right) / \varepsilon} .
$$

Let $\Psi_{2}$ be the solution of

$$
\left\{\begin{array}{l}
-\varepsilon^{2} \Delta \Psi+4 \gamma_{0} \Psi=0, \\
\Psi(x) \rightarrow 0 \text { as }|x| \rightarrow \infty, \\
\Psi=1 \quad \text { for } x \in \partial B_{\delta(1-\theta)}(y) .
\end{array}\right.
$$

We have

$$
0<\Psi_{2}(x) \leq C e^{-3 \gamma_{0} \operatorname{dist}\left(x, B_{\delta(1-\theta)}(y)\right) / \varepsilon} .
$$

Denote $a_{\varepsilon}(x)=V(x)-p\left(\Phi_{\varepsilon}(x)+U_{\varepsilon, y}\right)^{p-1}$. Then

$$
a_{\varepsilon}(x) \geq 6 \gamma_{0}-o(1)>5 \gamma_{0} \quad \text { for } x \in \mathbb{R}^{N} \backslash\left(\mathcal{Z}^{\delta} \cup B_{\delta(1-\theta)}(y)\right) .
$$

Setting $\Psi=\Psi_{1}+\Psi_{2}+e^{-(1+5 \mu) \sigma / \varepsilon}$ we have from (2.51)-(2.53) that for $x \in$ $\mathbb{R}^{N} \backslash\left(\mathcal{Z}^{\delta} \cup B_{\delta(1-\theta)}(y)\right)$

$$
-\varepsilon^{2} \Delta \Psi+a_{\varepsilon}(x) \Psi \geq-\varepsilon^{2} \Delta \Psi+5 \gamma_{0} \Psi \geq \gamma_{0} e^{-(1+5 \mu) \sigma / \varepsilon} \geq\left|f_{\varepsilon}\right| .
$$


From step 2 we have $\left|\omega_{1}(x)\right| \leq C \varepsilon^{\frac{\sigma}{2}}<\Psi(x)$ for $x \in \partial\left(\mathcal{Z}^{\delta} \cup B_{\delta(1-\theta)}(y)\right)$. Thus by maximum principle for $x \in \mathbb{R}^{N} \backslash\left(\mathcal{Z}^{\delta} \cup B_{\delta(1-\theta)}(y)\right)$ we have

$$
\left|\omega_{1}(x)\right| \leq \Psi(x)
$$

Therefore

$$
\left|\omega_{1}(x)\right| \leq C e^{-(1+5 \mu) \sigma / \varepsilon} \leq e^{-(1+4 \mu) \sigma / \varepsilon}, \quad \text { for } x \in \mathbb{R}^{N} \backslash\left(\mathcal{Z}^{\delta} \cup B_{\delta(1-\theta)}(y)\right) .
$$

In particular we have

$$
\left|\omega_{1}(x)\right| \leq e^{-(1+4 \mu) \sigma / \varepsilon} \quad \text { for } x \in \mathcal{Z}^{3 \delta} \backslash \mathcal{Z}^{\frac{5}{4} \delta} .
$$

Next we show that (2.54) still holds even if $x \in \mathcal{Z}^{\delta}$. To this end, let $\Psi_{3}$ be the solution of

$$
\begin{cases}-\Delta \Psi_{3}=0 & x \in \mathcal{Z}^{2 \delta} \\ \Psi_{3}(x)=\omega_{1}(x) & \text { for } x \in \partial \mathcal{Z}^{2 \delta} .\end{cases}
$$

Then it follows from maximum principle and (2.55) that

$$
\Psi_{3}(x) \leq e^{-(1+4 \mu) \sigma / \varepsilon} \quad \text { for } x \in \mathcal{Z}^{2 \delta} .
$$

Let $\bar{\omega}_{1}(x)=\omega_{1}(x)-\Psi_{3}(x)$. Then

$$
\begin{aligned}
& -\Delta \bar{\omega}_{1}+\varepsilon^{-2} V(x) \bar{\omega}_{1}-p \varepsilon^{-2}\left(\Phi_{\varepsilon}+U_{\varepsilon, y}\right)^{p-1} \bar{\omega}_{1} \\
& =\varepsilon^{-2} f_{\varepsilon}(x)-\varepsilon^{-2} V(x) \Psi_{3}(x)+p \varepsilon^{-2}\left(\Phi_{\varepsilon}+U_{\varepsilon, y}\right)^{p-1} \Psi_{3}(x) \\
& =\varepsilon^{-2} f_{\varepsilon}(x)+O\left(e^{-(1+3 \mu) \sigma / \varepsilon}\right) .
\end{aligned}
$$

We claim that

$$
\int_{\mathcal{Z}^{2 \delta}}\left(\left|\nabla \bar{\omega}_{1}\right|^{2}+\bar{\omega}_{1}^{2}\right) \leq C e^{-(1+3 \mu) \sigma / \varepsilon}
$$

Indeed, since for $\bar{\omega}_{1} \in H_{0}^{1}\left(\widehat{\Omega}^{2 \delta}\right)$, it follows from Proposition 2.4 and the estimate of $f_{\varepsilon}$ and (2.56) that

$$
\int_{\widehat{\Omega}^{2 \delta}}\left|\nabla \bar{\omega}_{1}\right|^{2} \leq \int_{\widehat{\Omega}^{2 \delta}}\left(\left|\nabla \bar{\omega}_{1}\right|^{2}+\varepsilon^{-2} V(x) \bar{\omega}_{1}^{2}\right) \leq C e^{-(1+3 \mu) \sigma / \varepsilon},
$$

which implies

$$
\int_{\widehat{\Omega}^{2 \delta}}\left(\left|\nabla \bar{\omega}_{1}\right|^{2}+\bar{\omega}_{1}^{2}\right) \leq C e^{-(1+3 \mu) \sigma / \varepsilon},
$$

since $\bar{\omega}_{1} \in H_{0}^{1}\left(\widehat{\Omega}^{2 \delta}\right)$ and $\widehat{\Omega}^{2 \delta}$ is bounded.

On the other hand, since the coefficient of the last term in the left hand side of (2.56), $p \varepsilon^{-2}\left(\Phi_{\varepsilon}+U_{\varepsilon, y}\right)$, is exponentially small for $x \in \mathcal{Z}^{2 \delta} \backslash \widehat{\Omega}^{2 \delta}$, from the estimate of $f_{\varepsilon}$ and (2.56) we have

$$
\int_{\mathcal{Z}^{2 \delta} \backslash \widehat{\Omega}^{2 \delta}}\left(\left|\nabla \bar{\omega}_{1}\right|^{2}+\bar{\omega}_{1}^{2}\right) \leq C e^{-(1+3 \mu) \sigma / \varepsilon}
$$

and (2.57) therefore follows.

Rewrite (2.56) as

$$
-\Delta \bar{\omega}_{1}=\bar{f}_{\varepsilon}(x),
$$

where

$$
\bar{f}_{\varepsilon}(x)=-\varepsilon^{-2} V(x) \bar{\omega}_{1}+p \varepsilon^{-2}\left(\Phi_{\varepsilon}+U_{\varepsilon, y}\right)^{p-1} \bar{\omega}_{1}+\varepsilon^{-2} f_{\varepsilon}(x)+O\left(e^{-(1+3 \mu) \sigma / \varepsilon}\right) .
$$


Using the Moser iterative technique (see Gilbarg and Trudinger [19]), we have

$$
\begin{aligned}
& \left\|\bar{\omega}_{1}\right\|_{L^{\infty}\left(\mathcal{Z}^{\frac{3}{2} \delta}\right)} \leq C\left(\left\|\bar{\omega}_{1}\right\|_{L^{2}\left(\mathcal{Z}^{2 \delta}\right)}+\left\|\bar{f}_{\varepsilon}\right\|_{L^{2}\left(\mathcal{Z}^{2 \delta}\right)}\right) \leq C \varepsilon^{-2} e^{-(1+3 \mu) \sigma / \varepsilon} \\
\leq & C e^{-(1+2 \mu) \sigma / \varepsilon},
\end{aligned}
$$

which, combined with (2.55), gives

$$
\left\|\omega_{1}\right\|_{L^{\infty}\left(\mathcal{Z}^{2 \delta}\right)} \leq C e^{-(1+2 \mu) \sigma / \varepsilon} .
$$

Thus, we complete step 3 .

Step 4. $\omega_{1} \in S_{\varepsilon}$.

By step 1, step 2 and step 3 , we only need to obtain the estimate for $\omega_{1}(x)$ when $x \in B_{\delta}(y)$.

For $x \in B_{\delta}(y)$, we have

$$
|\omega(x)|^{\bar{p}} \leq C e^{-\bar{p} \sigma|x-y| /(\varepsilon \delta)} \varepsilon^{\sigma \bar{p}}
$$

and

$$
\begin{aligned}
\left|V(x)-V\left(a_{1}\right)\right| U\left(\frac{x-y}{\varepsilon}\right) & \leq|V(x)-V(y)| U\left(\frac{x-y}{\varepsilon}\right)+\left|V(y)-V\left(a_{1}\right)\right| U\left(\frac{x-y}{\varepsilon}\right) \\
& \leq C|x-y| U\left(\frac{x-y}{\varepsilon}\right)+C \varepsilon^{2} U\left(\frac{x-y}{\varepsilon}\right) \\
& \leq C \varepsilon^{(1+\mu) \sigma} e^{-\sigma|x-y| /(\varepsilon \delta)}
\end{aligned}
$$

provided $\sigma$ is small so that $\sigma<\frac{1}{2} \delta \sqrt{V\left(x_{0}\right)}$. By step 2, for $x \in B_{\delta}(y)$,

$$
\begin{aligned}
\left(\Phi_{\varepsilon}+U_{\varepsilon, y}\right)^{p-1}\left|\omega_{1}\right| & \leq C\left(W_{\varepsilon}^{p-1}(x)+\widetilde{W}_{\varepsilon}^{p-1}(x)+U^{p-1}\left(\frac{x-y}{\varepsilon}\right)\right)\left|\omega_{1}(x)\right| \\
& \leq C \varepsilon^{(1+\mu) \sigma} e^{-\sigma|x-y| /(\varepsilon \delta)}
\end{aligned}
$$

if $\sigma$ is small by (1.9), (1.10).

Let $\tilde{f}_{\varepsilon}(x)=f_{\varepsilon}(x)+p\left(\Phi_{\varepsilon}(x)+U_{\varepsilon, y}(x)\right)^{p-1} \omega_{1}(x)$. Then

$$
-\varepsilon^{2} \Delta \omega_{1}+V(x) \omega_{1}=\tilde{f}_{\varepsilon}(x) .
$$

By (2.59)-(2.61) we have

$$
\left|\tilde{f}_{\varepsilon}(x)\right| \leq C \varepsilon^{(1+\mu) \sigma} e^{-\sigma|x-y| /(\varepsilon \delta)} \quad \forall x \in B_{\delta}(y) .
$$

On the other hand, let $\Psi_{4}(x)=\varepsilon^{\sigma} e^{-\sigma|x-y| /(\varepsilon \delta)}$. Then we have

$$
-\varepsilon^{2} \Delta \Psi_{4}+V(x) \Psi_{4} \geq \frac{1}{2} V\left(x_{0}\right) \varepsilon^{\sigma} e^{-\sigma|x-y| /(\varepsilon \delta)} \geq\left|\bar{f}_{\varepsilon}(x)\right| .
$$

Notice that by step 3 ,

$$
\left|\omega_{1}(x)\right| \leq C e^{-(1+\mu) \sigma / \varepsilon} \leq \Psi_{4}(x) \quad \text { for } \quad x \in \partial B_{\delta}(y) .
$$

By maximum principle we obtain from (2.62), (2.64) and (2.65)

$$
\left|\omega_{1}(x)\right| \leq \Psi_{4}(x)=\varepsilon^{\sigma} e^{-\sigma|x-y| /(\varepsilon \delta)} \quad \text { for } \quad x \in B_{\delta}(y),
$$

which is exactly what we want. Hence step 4 is completed.

Thus, $G_{\varepsilon}(\omega)$ is a contraction mapping from $\mathcal{S}_{\varepsilon}$ to itself. Therefore, by the contraction mapping theorem, $G_{\varepsilon}(\omega)$ has a fixed point $\omega_{\varepsilon} \in \mathcal{S}_{\varepsilon}$. As a result,

$$
\omega_{\ell_{\varepsilon}}+L_{\varepsilon} \omega_{\varepsilon}+R_{\varepsilon}^{\prime}\left(\omega_{\varepsilon}\right)=0 .
$$

Consequently, there exist $D_{j, \varepsilon} \in \mathbb{R}$ for $j=1, \cdots, N$ such that

$$
I^{\prime}\left(U_{\varepsilon, y}+\omega_{\varepsilon}\right)=\sum_{j=1}^{N} D_{j, \varepsilon} \frac{\partial U_{\varepsilon, y}}{\partial x_{j}} .
$$


Moreover, by step 1 and step 2, we have

$$
\left\{\begin{array}{l}
\left\|\omega_{\varepsilon}\right\|_{\varepsilon}=O\left(\varepsilon^{\frac{N}{2}+2}\right), \\
D_{i, \varepsilon}=O\left(\varepsilon^{3}\right) .
\end{array}\right.
$$

This completes our proof of Proposition 2.5.

\section{Proof of the main Result}

In this section we will give the proof of Theorem 1.3 . The proof of Theorem 1.1 is similar. Let us point out that in the case $l=0$, the result follows directly from Proposition 2.3 and the implicit function theorem.

Proof of Theorem 1.3. We need to prove that there is $y_{\varepsilon} \in B_{\delta}\left(a_{1}\right)$ such that (1.23) holds.

Direct calculations show that

$$
\begin{aligned}
& \frac{\partial J}{\partial y_{j}} \\
= & \left\langle U_{\varepsilon, y}+\omega_{\varepsilon}, \frac{\partial U_{\varepsilon, y}}{\partial y_{j}}\right\rangle-\int_{\mathbb{R}^{N}}\left(U_{\varepsilon, y}+\omega_{\varepsilon}\right)_{+}^{p} \frac{\partial U_{\varepsilon, y}}{\partial y_{j}}-\int_{\mathbb{R}^{N}} g_{\varepsilon}\left(x, U_{\varepsilon, y}+\omega_{\varepsilon}\right) \frac{\partial U_{\varepsilon, y}}{\partial y_{j}} \\
= & \int_{\mathbb{R}^{N}} V(x) U_{\varepsilon, y} \frac{\partial U_{\varepsilon, y}}{\partial y_{j}}-\int_{\mathbb{R}^{N}}\left(\left(U_{\varepsilon, y}+\omega_{\varepsilon}\right)_{+}^{p}-U_{\varepsilon, y}^{p}-p U_{\varepsilon, y}^{p-1} \omega_{\varepsilon}\right) \frac{\partial U_{\varepsilon, y}}{\partial y_{j}} \\
& -\int_{\mathbb{R}^{N}} g_{\varepsilon}\left(x, U_{\varepsilon, y}+\omega_{\varepsilon}\right) \frac{\partial U_{\varepsilon, y}}{\partial y_{j}} \\
= & \int_{\mathbb{R}^{N}} V(x) U_{\varepsilon, y} \frac{\partial U_{\varepsilon, y}}{\partial y_{j}}+O\left(e^{-\gamma / \varepsilon}+\varepsilon^{-1}\left\|\omega_{\varepsilon}\right\|_{\varepsilon}^{2}\right) \\
= & \frac{1}{2} \int_{\mathbb{R}^{N}} U_{\varepsilon, y}^{2} \frac{\partial V}{\partial x_{j}}+O\left(\varepsilon^{N+3}\right) \\
= & \varepsilon^{N} \frac{\partial V(y)}{\partial y_{j}} \frac{1}{2} \int_{\mathbb{R}^{N}} U^{2}+O\left(\varepsilon^{N+1+\alpha}\right),
\end{aligned}
$$

since

$$
\int_{\mathbb{R}^{N}} U_{\varepsilon, y}^{2}\left(x_{i}-y_{i}\right) d x=0
$$

By step 2 in Proposition 2.5, we have

$$
D_{j, \varepsilon}=O\left(\varepsilon^{3}\right) .
$$

So, it is easy to see that (1.23) is equivalent to

$$
\nabla V(y)=O\left(\varepsilon^{1+\alpha}\right)
$$

Since $a_{1}$ is a nondegenerate critical point of $V(x)$, we see that the above relation is equivalent to

$$
y-a_{1}=h_{\varepsilon}(y),
$$

where $h_{\varepsilon}(y)$ satisfies $\left|h_{\varepsilon}(y)\right| \leq C \varepsilon^{1+\alpha}$ for all $y \in B_{\varepsilon}\left(a_{1}\right)$. Thus, $h_{\varepsilon}$ maps $B_{\varepsilon}\left(a_{1}\right)$ into itself. By the fixed point theorem, we deduce that there is a $y_{\varepsilon} \in B_{\varepsilon}\left(a_{1}\right)$ such that (1.23) holds. 
Therefore $U\left(\frac{x-y_{\varepsilon}}{\varepsilon}\right)+\omega_{\varepsilon}$ is a critical point of $I$ in $H^{1}\left(\mathbb{R}^{N}\right)$, which yields that $u_{\varepsilon}(x)=W_{\varepsilon}(x)+\widetilde{W}_{\varepsilon}(x)+U\left(\frac{x-y_{\varepsilon}}{\varepsilon}\right)+\omega_{\varepsilon}$ is a solution of

$$
\begin{cases}-\varepsilon^{2} \Delta u+V(x) u=u_{+}^{p}, & x \in \mathbb{R}^{N}, \\ u(x) \rightarrow 0, & \text { as }|x| \rightarrow \infty .\end{cases}
$$

By the maximum principle, we see that $u_{\varepsilon}>0$.

We can also use the above techniques to deal with some degenerate case. We have

Theorem 3.1. Suppose that $k=t=l=1$. Assume (V1), (V2), and (V3) hold. Suppose that $a_{1}$ is an isolated strict local maximum point or minimum point of $V(x)$ with $V\left(a_{1}\right)>0$. Then for $\varepsilon>0$ small, (1.6) has a solution $u_{\varepsilon}$ such that

$$
u_{\varepsilon}(x)=W_{\varepsilon}(x)+\widetilde{W}_{\varepsilon}(x)+U\left(\frac{x-y_{\varepsilon}}{\varepsilon}\right)+\omega_{\varepsilon}(x)
$$

where $\omega_{\varepsilon}(x)$ and $y_{\varepsilon}$ satisfy (1.17), (1.18) and (1.19) in Theorem 1.1.

Proof. First, we need to modify the proof of Proposition 2.5. Define

$$
\mathcal{M}_{\varepsilon}=\left\{y \in B_{\delta}\left(a_{1}\right)|\quad| V(y)-V\left(a_{1}\right) \mid \leq \varepsilon^{(1+\tau) \sigma}\right\},
$$

where $\tau>0$ and $\sigma>0$ are some small constants. Then, similar to the proof of Proposition 2.5. we can prove that for each $y \in \mathcal{M}_{\varepsilon}$, there is an $\omega_{\varepsilon} \in \mathcal{S}_{\varepsilon}$ such that (2.31) holds and

$$
\left\|\omega_{\varepsilon}\right\|_{\varepsilon}=O\left(\varepsilon^{(1+\tau) \sigma}\right) .
$$

Define

$$
K(y)=J\left(y, \omega_{\varepsilon}\right),
$$

where $\omega_{\varepsilon}$ is the function obtained above.

It follows from (2.2), Lemmas 2.1 and 2.2 that

$$
\begin{aligned}
K(y) & =J(y, 0)+O\left(\left\|\ell_{\varepsilon}\right\|_{\varepsilon}\left\|\omega_{\varepsilon}\right\|_{\varepsilon}+\left\|\omega_{\varepsilon}\right\|_{\varepsilon}^{2}\right) \\
& =I\left(U_{\varepsilon, y}\right)+O\left(\varepsilon^{N+2(1+\tau) \sigma}\right) .
\end{aligned}
$$

On the other hand, by the definition of $g_{\varepsilon}(x, t)$, it is easy to see

$$
\left|G_{\varepsilon}(x, t)\right| \leq C\left(\widetilde{W}_{\varepsilon}+W_{\varepsilon}\right)\left(|t|+|t|^{p}\right) .
$$

Since $\widetilde{W}_{\varepsilon}$ and $W_{\varepsilon}$ are exponentially small near $y$ and $U_{\varepsilon, y}$ is exponentially small outside a small neighborhood of $y$, we obtain

$$
\left|\int_{\mathbb{R}^{N}} G\left(x, U_{\varepsilon, y}\right)\right| \leq C e^{-\sigma^{\prime} / \varepsilon}
$$

for some $\sigma^{\prime}>0$.

Combining (3.2) and (3.3), we obtain

$$
K(y)=I^{*}\left(U_{\varepsilon, y}\right)+O\left(\varepsilon^{N+2(1+\tau) \sigma}\right),
$$

where

$$
I^{*}(u)=\frac{1}{2} \int_{\mathbb{R}^{N}}\left(\varepsilon^{2}|\nabla u|^{2}+V(x) u^{2}\right)-\frac{1}{p+1} \int_{\mathbb{R}^{N}} u_{+}^{p+1} .
$$

Standard calculations show that

$$
I^{*}\left(U_{\varepsilon, y}\right)=A \varepsilon^{N}+B\left(V(y)-V\left(a_{1}\right)\right) \varepsilon^{N}+O\left(\varepsilon^{N+2}\right),
$$

where $A=\left(\frac{1}{2}-\frac{1}{p+1}\right) \int_{\mathbb{R}^{N}} U^{p+1}, B=\frac{1}{2} \int_{\mathbb{R}^{N}} U^{2}$. 
Combining (3.4) and (3.5), we obtain

$$
K(y)=A \varepsilon^{N}+B\left(V(y)-V\left(a_{1}\right)\right) \varepsilon^{N}+O\left(\varepsilon^{N+2(1+\tau) \sigma}\right) .
$$

Let us first consider the case that $a_{1}$ is a strict local maximum point. Consider

$$
K_{\max }=\max \left\{K(y) \mid y \in \mathcal{M}_{\varepsilon}\right\} .
$$

Suppose that $y_{\varepsilon} \in \mathcal{M}_{\varepsilon}$ achieves $K_{\max }$. We will prove that $y_{\varepsilon}$ is an interior point of $\mathcal{M}_{\varepsilon}$. So $y_{\varepsilon}$ is a critical point of $K(y)$.

We have

$$
K\left(y_{\varepsilon}\right) \geq K\left(a_{1}\right)=A \varepsilon^{N}+O\left(\varepsilon^{N+2(1+\tau) \sigma}\right),
$$

which, together with (3.6), gives

$$
A \varepsilon^{N}+B\left(V\left(y_{\varepsilon}\right)-V\left(a_{1}\right)\right) \varepsilon^{N}+O\left(\varepsilon^{N+2(1+\tau) \sigma}\right) \geq A \varepsilon^{N}+O\left(\varepsilon^{N+2(1+\tau) \sigma}\right) .
$$

As a result,

$$
0 \leq V\left(a_{1}\right)-V\left(y_{\varepsilon}\right) \leq C \varepsilon^{2(1+\tau) \sigma}<\varepsilon^{(1+\tau) \sigma} .
$$

This shows that $y_{\varepsilon}$ is an interior point of $\mathcal{M}_{\varepsilon}$. Standard argument shows that (1.23) holds.

Suppose that $a_{1}$ is a strict local minimum point. Consider

$$
K_{\min }=\min \left\{K(y) \mid y \in \mathcal{M}_{\varepsilon}\right\} .
$$

Suppose that $\tilde{y}_{\varepsilon}$ achieves $K_{\min }$. Then, similar to the above argument, we can prove that $\tilde{y}_{\varepsilon}$ is an interior point if $\mathcal{M}_{\varepsilon}$, and thus a critical point of $K(y)$.

\section{ACKNOWLEDGEMENTS}

The authors are grateful for the anonymous referee for very helpful suggestions and comments.

\section{REFERENCES}

[1] A. Ambrosetti, A. Malchoidi and S. Secchi, Multiplicity results for some nonlinear Schrödinger equations with potentials, Arch. Rat. Mech. Anal., 159(2001), 253-271. MR1857674 (2002m:35044)

[2] A. Ambrosetti, M. Badiale and S. Cingolani, Semiclassical states of nonlinear Schrödinger equations, Arch. Rat. Mech. Anal., 140(1997), 285-300. MR1486895 (98k:35172)

[3] T. Bartsch and Z.-Q. Wang, Multiple positive solutions for a nonlinear Schrödinger equation, Z. Angew. Math. Phys., 51(2000), 366-384. MR1762697 (2001f:35363)

[4] J. Byeon and Z.-Q. Wang, Standing waves with a critical frequency for nonlinear Schrödinger equations, Arch. Rat. Mech. Anal., 165(2002), 295-316. MR1939214 (2003i:35250)

[5] J. Byeon and Z.-Q. Wang, Standing waves with a critical frequency for nonlinear Schrödinger equations, II, Calc. Var. and PDE., 18(2003), 207-219. MR2010966 (2004h:35207)

[6] J. Byeon and Y. Oshita, Existence of multi-bump standing waves with a critical frequency for nonlinear Schrödinger equations, Comm. in P.D.E., 29(2004), 1877-1904. MR2106071 (2006e:35084)

[7] J. Byeon and Y. Oshita, Uniqueness of standing waves for nonlinear Schrödinger equations, preprint.

[8] D. Cao and H. P. Heinz, Uniqueness of positive multi-lump bounded states of nonlinear Schrödinger equations, Math. Z., 243(2003), 599-642. MR1970017 (2004m:35244)

[9] D. Cao and E. S. Noussair, Multi-bump standing waves with a critical frequency for nonlinear Schrödinger equations, J. Diff. Equats., 203(2004), 292-312. MR2073688 (2005g:35265)

[10] D. Cao, E. Noussair and S. Yan, Solutions with multiple peaks for nonlinear elliptic equations, Proc. Roy. Soc. Edinburgh, A129(1999), 235-264. MR.1686700(2000a:35052) 
[11] D. Cao, E. Noussair and S. Yan, Existence and uniqueness results on single-peaked solutions of a semilinear problem, Ann. Inst. H. Poincaré, Anal. non Linéaire, 15(1998), 73-111. MR:1614607 (99d:35053)

[12] S. Cingolani and M. Lazzo, Multiple positive solutions to nonlinear Schrödinger equations with competing potential functions, J. Diff. Equats., 160(2000), 118-138. MR1734531 (2000j:35079)

[13] E. N. Dancer and S. Yan, On the existence of multi peak solutions for nonlinear field equations on $R^{N}$, Discrete and Continuous Dynamic Systems, 6(2000), 39-50. MR1739592 (2000k:35081)

[14] M. Del Pino and M. Felmer, Local mountain passes for semilinear elliptic problems in unbounded domains, Calc. Var. and PDE., 4(1996), 121-137. MR1379196 (97c:35057)

[15] M. Del Pino and M. Felmer, Semi-classical states for nonlinear Schrödinger equations, J. Funct. Anal., 149(1997), 245-265. MR1471107(98i:35183)

[16] M. Del Pino and M. Felmer, Multi-peak bound states for nonlinear Schrödinger equations, Ann. Inst. H. Poincaré, Anal. non Linéaire, 15(1998), 127-149. MR1614646 (99c:35228)

[17] M. Del Pino and M. Felmer, Semi-classical states of nonlinear Schrödinger equations: a variational reduction method, Math. Ann., 324(2002), 1-32. MR1931757 (2003g:35081)

[18] A. Floer and A. Weinstein, Non spreading wave packets for the cubic Schrödinger equations with a bounded potential, J. Funct. Anal., 69(1986), 397-408. MR867665 (88d:35169)

[19] D. Gilbarg and N. S. Trudinger, Elliptic Partial Differential Equations of Second Order, Second edition. Grundlehren 224, Springer, Berlin, Heidelberg, New York and Tokyo, 1983. MR.737190 (86c:35035)

[20] M. Grossi, On the number of single-peak solutions of the nonlinear Schrödinger equation, Ann. I. H. Poincaré, Anal. non linéaire, 19(2002), 261-280. MR.1956951 (2003k:35228)

[21] M. Grossi, A uniqueness result for a semilinear elliptic equation in symmetric domains, Adv. Diff. Equats., 5(2000), 193-212. MR1734541(2001a:35065)

[22] C. Gui, Existence of multi-bump solutions for nonlinear Schrödinger equations via variational method, Comm. in P.D.E., 21(1996), 787-820. MR1391524 (98a:35122)

[23] M. Hirose and M. Ohta, Structure of positive solutions to scalar field equations with harmonic potential, J.Diff. Equats., 178(2002), 519-540. MR1879836 (2002k:35089)

[24] X. Kang and J. Wei, On interacting bumps of semi-classical states of nonlinear Schrödinger equations, Adv. Diff. Equats., 5(2000), 899-928. MR1776345 (2001k:35093)

[25] Y. Y. Li, On a singular perturbed elliptic equation, Adv. Diff. Equats., 2(1997), 955-980. MR:1606351 (99b:35005)

[26] W.-M. Ni and I. Takagi, On the shape of least-energy solutions to a semilinear Neumann problem, Comm. Pure Appl. Math., 44(1991), 819-851. MR.1115095 (92i:35052)

[27] E. S. Noussair and S. Yan, On positive multipeak solutions for a nonlinear elliptic problem, J. London Math. Soc., 62(2000), 213-227. MR1772182 (2001k:35098)

[28] Y. G. Oh, Existence of semi-classical bound states of nonlinear Schrödinger equations with potential on the class $(V)_{\alpha}$, Comm. in P.D.E., 13(1988), 1499-1519. MR970154(90d:35063a)

[29] Y. G. Oh, On positive multi-lump bound states of nonlinear Schrödinger equations under multiple well potential, Comm. Math. Phys., 131(1990), 223-253. MR.1065671(92a:35148)

[30] P. H. Rabinowitz, On a class of nonlinear Schrödinger equations, Z. Angew. Math. Phys., 43(1992), 270-291. MR1162728 (93h:35194)

[31] B. Sirakov, Standing waves solutions of the nonlinear Schrödinger equation in $\mathbf{R}^{N}$, Ann. Mat. Pura Appl., 181(2002), 73-83. MR1895026 (2003a:35180)

[32] Z. Q. Wang, Existence and symmetry of multi-bump solutions for nonlinear Schrödinger equations, J. Diff. Equats., 159(1999), 102-137. MR:1726920(2001h:35176)

Institute of Applied Mathematics, AMSS, Chinese Academy of Sciences, Beijing 100080, People's Republic of China

E-mail address: dmcao@amt.ac.cn

School of Mathematics, The University of New South Wales, Sydney 2052, Australia

E-mail address: noussair@maths.unsw.edu.au

School of Mathematics, Statistics and Computer Science, The University of New England, Armidale NSW 2351, Australia

E-mail address: syan@turing.une.edu.au 